\title{
Finite-time anti-synchronization of multi-weighted coupled neural networks with and without coupling delays
}

\author{
Jie Hou • Yanli Huang · Erfu Yang
}

Received: date / Accepted: date

\begin{abstract}
The multi-weighted coupled neural networks (MWCNNs) models with and without coupling delays are investigated in this paper. Firstly, the finite-time anti-synchronization of MWCNNs with fixed topology and switching topology is analyzed respectively by utilizing Lyapunov functional approach as well as some inequality techniques, and several anti-synchronization criteria are put forward for the considered networks. Furthermore, when the parameter uncertainties appear in MWCNNs, some conditions for ensuring robust finite-time anti-synchronization are obtained. Similarly, we also consider the finite-time anti-synchronization and robust finite-time anti-synchronization for MWCNNs with coupling delays under fixed and switched topologies respectively. Lastly, two numerical examples with simulations are provided to confirm the effectiveness of these derived results.
\end{abstract}

Keywords Finite-time anti-synchronization - Coupled neural networks · Multiple weights · Robust anti-synchronization · Switching topology

\section{Introduction}

In recent years, neural networks (NNs) have become a hot topic because of their widespread applications, especially in optimization, associate memory,

Jie Hou

School of Computer Science and Technology, Tianjin Polytechnic University, Tianjin 300387, China

Yanli Huang (Corresponding author)

Tianjin Key Laboratory of Optoelectronic Detection Technology and System, School of Computer Science and Technology, Tianjin Polytechnic University, Tianjin 300387, China E-mail: huangyanli@tjpu.edu.cn

Erfu Yang

Department of Design, Manufacture and Engineering Management, Faculty of Engineering, University of Strathclyde, Glasgow G1 1XJ, Scotland, UK 
pattern recognition and so on [1-7]. Actually, these applications are heavily dependent on their dynamical behaviors. Hence, a lot of meaningful and important research results have been reported on stability and passivity of NNs [8-13]. Zhang et al. [8] studied the stability for a class of discrete-time NNs with time-varying delay via an extended reciprocally convex matrix inequality. The authors in [9] discussed the stability of complex-valued memristive recurrent NNs. By utilizing Lyapunov functional approach and some inequality techniques, the passivity and stability problems for $\mathrm{NNs}$ with reaction-diffusion terms were considered in [10].

As a particular sort of complex networks, coupled NNs (CNNs) is composed of many NNs which interact with each other. As we all known, synchronization is one of the most significant dynamic properties in CNNs, which has been applied in many areas such as image processing, secure communication $[14,15]$. Therefore, it is of great significance to investigate the synchronization of CNNs [16-22]. Wang et al. [18] studied the local synchronization problem for a sort of Markovian nonlinearly CNNs by applying the LyapunovKrasovskii functional as well as free-matrix-based integral inequality. Based on Lyapunov functions, Halanay inequality and stochastic analysis technique, the exponential synchronization problem of CNNs with time-varying delay was investigated in [19]. Several criteria for ensuring exponential synchronization for markovian stochastic CNNs were carried out via adaptive feedback control in [20]. The work in [22] analyzed the exponential synchronization and passivity of CNNs with reaction-diffusion terms by adopting appropriate pinning controllers. What is noteworthy is that anti-synchronization is also a fascinating phenomenon in the real world, which widely exists in memristive recurrent NNs, periodic oscillators, and so forth. Moreover, up to now, anti-synchronization has been successfully applied in many fields, for instance, image processing, information science and so on. Hence, it is highly meaningful to study anti-synchronization [23-28]. Some conditions for guaranteeing anti-synchronization of CNNs with mixed time-varying delays were set up via randomly occurring control in [25]. Liu et al. [26] devoted themselves to the anti-synchronization for the considered complex-valued memristive NNs by employing suitable Lyapunov functional and some inequality techniques. In [28], the anti-synchronization problem of a memristor-based bidirectional associate memory NN was studied by utilizing a robust feedback controller with an appropriate gain control matrix.

In many practical applications, it is usually required to realize synchronization within a limited time interval. Accordingly, a large number of literatures on finite-time synchronization have been reported [29-40]. In [29], several conditions for ensuring the finite-time synchronization of CNNs with time-varying delays were derived by designing a stochastic multiple Lyapunov-Krasovskii function and using weighted integral inequality. Some discontinuous or continuous controllers were constructed for the finite-time synchronization problem of switched CNNs in [30]. Sun et al. [32] investigated the finite-time synchronization for two complex-variable chaotic systems with unknown parameters via nonsingular terminal sliding mode control. However, only a few results have 
been obtained about finite-time anti-synchronizaton up to now [41, 42]. In [41], the finite-time anti-synchronization for a class of time-varying delayed NNs was studied via feedback control with intermittent adjustment. The authors proposed some finite-time anti-synchronization criteria for memristive NNs by designing a nonlinear controller in [42]. As far as we know, a great many of existing networks can be described more accurately by multi-weighted complex dynamical networks (MWCDNs), for instance, transportation networks, social networks, communication networks and so on. Therefore, it is of great significance to study MWCDNs [43]. Qiu et al. [43] proposed several finite-time synchronization conditions for MWCDNs with and without coupling delays by means of Lyapunov functional approach and state feedback controllers. However, there are only a few results reported on the finite-time synchronization of MWCNNs [44]. In [44], several novel criteria for guaranteeing finite-time synchronization of MWCNNs with and without coupling delays were provided by exploiting new definitions of finite-time synchronization and designing appropriate controllers. It is a pity that the finite-time anti-synchronization of MWCNNs has not yet been considered in existing research results.

As is well known, the connection topology of most of networks mentioned above is always supposed to be fixed. Nevertheless, in practical applications, this requirement is very strict because of the impacts of limited communications and external disturbances. Therefore, more and more researchers devoted themselves to studying the synchronization of NNs with switching topology [45-48]. In [46], the authors investigated the finite-time synchronization of a sort of uncertain CNNs with switching topology by constructing appropriate Lyapunov-like functionals and using the average dwell time technique. The work in [47] proposed some conditions for guaranteeing pinning synchronization of directed networks with switching topologies by employing a multiple lyapunov functions approach. Based on Lyapunov functional approach and some inequality techniques, Qin et al. [48] studied the synchronization and $\mathcal{H}_{\infty}$ synchronization of MWCDNs with fixed and switching topologies. However, there is no research results reported on the finite-time anti-synchronization for MWCNNs with switching topology until now.

On the other hand, some uncertain factors may be occurred in NNs due to the existence of environmental noises and modeling errors, which might cause the exact parameter values of NNs could not be obtained. Therefore, it is important to consider the robust synchronization of CNNs which consisting of several identical uncertain NNs [49-52]. In [49], the authors investigated the robust synchronization of fractional-order CNNs by utilizing pinning control strategies. The robust synchronization problem for delayed CNNs with uncertain parameters was studied by intermittent pinning control in [50]. By using inequality techniques and constructing appropriate Lyapunov functional, Qin et al. [51] discussed the robust synchronization and $\mathcal{H}_{\infty}$ synchronization of MWCDNs with uncertain parameters. To our best knowledge, the robust finite-time anti-synchronization for MWCNNs with uncertain parameters has never been discussed. 
On the basis of the above discussion, we study the finite-time anti-synchron ization and robust finite-time anti-synchronization of MWCNNs with fixed and switching topologies in this paper. Firstly, we present two MWCNN models. The first one is with constant coupling, and the second MWCNN is with delayed coupling. Then, several finite-time anti-synchronization criteria for these considered networks with fixed as well as switching topologies are derived respectively. Furthermore, some sufficient conditions for ensuring robust finitetime anti-synchronization of MWCNNs with fixed and switching topologies are also established.

In view of the above-mentioned discussion, the principal objective of this paper is to study the finite-time anti-synchronization of multi-weighted coupled neural networks (MWCNNs). The main contribution of this paper can be summarized as follows.

(1) The finite-time anti-synchronization of MWCNNs with fixed and switching topologies is studied and several criteria are put forward by designing suitable controller and constructing appropriate Lyapunov functional.

(2) Some conditions for guaranteeing the robust finite-time anti-synchronization of MWCNNs with uncertain parameters under fixed and switching topologies are established respectively.

(3) For the delayed MWCNNs, the finite-time anti-synchronization and robust finite-time anti-synchronization conditions are also derived similarly.

The outline of this paper is organized as follows. Several lemmas needed to be used throughout this paper are provided in Section 2. Section 3 is devoted to analyzing finite-time anti-synchronization and the robust finite-time anti-synchronization for MWCNNs with fixed and switching topologies, respectively. In Section 4, the network model of MWCNNs with coupling delays is introduced, and then the finite-time anti-synchronization and the robust finitetime anti-synchronization for this kind of MWCNNs with fixed and switching topologies are investigated. Two simulation examples are presented in Section 5 to verify the effectiveness of the obtained theoretical results. Finally, this paper is concluded in Section 6 .

\section{Preliminaries}

$0 \geqslant \chi \in \mathbb{R}^{n \times n}\left(0 \leqslant \chi \in \mathbb{R}^{n \times n}\right)$ symbols the matrix $\chi$ is semi-negative (semipositive) definite and symmetric, $0>\chi \in \mathbb{R}^{n \times n}\left(0<\chi \in \mathbb{R}^{n \times n}\right)$ shows that the matrix $\chi$ is negative (positive) definite and symmetric. $\lambda_{M}(\cdot)$ and $\lambda_{m}(\cdot)$ respectively signify the maximum and minimum eigenvalues of the corresponding matrix. In addition, for $e(t)=\left(e_{1}(t), e_{2}(t), \cdots, e_{n}(t)\right)^{T} \in \mathbb{R}^{n}$, we have $\|e(t)\|_{2}=\left(\sum_{\iota=1}^{n} e_{\iota}^{2}(t)\right)^{\frac{1}{2}}$.

Lemma 1 (see [53]). Suppose that a continuous and positive-definite function $H(t)$ meets the following inequality:

$$
\dot{H}(t) \leqslant-\mu(H(t))^{\omega}, t \geqslant 0, H(0) \geqslant 0,
$$


where $0<\mu \in \mathbb{R}$ and $0<\omega<1$ are constants. Then,

$$
(H(t))^{1-\omega} \leqslant(H(0))^{1-\omega}-\mu(1-\omega) t, 0 \leqslant t \leqslant T,
$$

and

$$
H(t)=0, t \geqslant T \text {, }
$$

with $T$ given by

$$
T=\frac{(H(0))^{1-\omega}}{\mu(1-\omega)} .
$$

Lemma 2 (see [54]). For $\nu_{\xi} \in \mathbb{R}, \xi=1,2, \cdots, n, 0<k \leqslant 1$, then

$$
\left|\nu_{1}\right|^{k}+\left|\nu_{2}\right|^{k}+\cdots+\left|\nu_{n}\right|^{k} \geqslant\left(\left|\nu_{1}\right|+\left|\nu_{2}\right|+\cdots+\left|\nu_{n}\right|\right)^{k} \text {. }
$$

\section{Finite-time anti-synchronization of MWCNNs}

3.1 Anti-synchronization in finite time for MWCNNs

\subsubsection{Anti-synchronization in finite time for MWCNNs with fixed topology}

Consider the following MWCNNs with fixed topology:

$$
\begin{aligned}
\dot{Y}_{\iota}(t)= & -A Y_{\iota}(t)+D g\left(Y_{\iota}(t)\right)+c_{1} \sum_{j=1}^{\kappa} G_{\iota j}^{1} \Gamma_{1} Y_{j}(t)+c_{2} \sum_{j=1}^{\kappa} G_{\iota j}^{2} \Gamma_{2} Y_{j}(t)+\cdots \\
& +c_{m} \sum_{j=1}^{\kappa} G_{\iota j}^{m} \Gamma_{m} Y_{j}(t)+u_{\iota}(t), \quad \iota=1,2, \cdots, \kappa
\end{aligned}
$$

where $Y_{\iota}(t)=\left(Y_{\iota 1}(t), Y_{\iota 2}(t), \cdots, Y_{\iota n}(t)\right)^{T} \in \mathbb{R}^{n}$ is the state vector of the $\iota$ th node; $A=\operatorname{diag}\left(a_{1}, a_{2}, \cdots, a_{n}\right) \in \mathbb{R}^{n \times n}>0, D=\left(d_{\iota j}\right)_{n \times n} \in \mathbb{R}^{n \times n}$ symbols a constant matrix; $g\left(Y_{\iota}(t)\right)=\left(g_{1}\left(Y_{\iota 1}(t)\right), g_{2}\left(Y_{\iota 2}(t)\right), \cdots, g_{n}\left(Y_{\iota n}(t)\right)\right)^{T} \in \mathbb{R}^{n}$ and $\mathbb{R} \ni c_{s}>0$ represents coupling strength for the $s$ th coupling form; $u_{\iota}(t) \in \mathbb{R}^{n}$ means the control input; $\Gamma_{s} \in \mathbb{R}^{n \times n}$ is the inner coupling matrix of the $s$ th coupling form; $G^{s}=\left(G_{\iota j}^{s}\right)_{\kappa \times \kappa} \in \mathbb{R}^{\kappa \times \kappa}$ expresses coupling weight between nodes in the $s$ th coupling form, where $G_{\iota j}^{s}$ is defined as follows: $G_{\iota j}^{s}=G_{j \iota}^{s}>0$ if and only if there exists a connection between node $\iota$ and node $j$ for the $s$ th coupling form; if not, $G_{\iota j}^{s}=G_{j \iota}^{s}=0(\iota \neq j)$; and

$$
G_{\iota \iota}^{s}=-\sum_{\substack{j=1 \\ j \neq \iota}}^{\kappa} G_{\iota j}^{s}, \iota=1,2, \cdots, \kappa,
$$

where $s=1,2, \cdots, m$.

Assumption 1 The function $g_{k}(\cdot)(k=1,2, \cdots, n)$ satisfies

$$
\left|g_{k}\left(\alpha_{1}\right)+g_{k}\left(\alpha_{2}\right)\right| \leqslant \theta_{k}\left|\alpha_{1}+\alpha_{2}\right|,
$$

for any $\alpha_{1}, \alpha_{2} \in \mathbb{R}$, where $0<\theta_{k} \in \mathbb{R}$. Take $\Theta=\operatorname{diag}\left(\theta_{1}^{2}, \theta_{2}^{2}, \cdots, \theta_{n}^{2}\right) \in \mathbb{R}^{n \times n}$. 
Suppose $Y_{0}(t)=\left(Y_{01}(t), Y_{02}(t), \cdots, Y_{0 n}(t)\right)^{T} \in \mathbb{R}^{n}$ is an arbitrary solution for the network (1), then

$$
\dot{Y}_{0}(t)=-A Y_{0}(t)+D g\left(Y_{0}(t)\right) \text {. }
$$

Definition 1 For all $\iota=1,2, \cdots, \kappa$, if there exists a constant $T>0$ such that

$$
\begin{aligned}
& \lim _{t \rightarrow T}\left\|Y_{\iota}(t)+Y_{0}(t)\right\|_{2}=0, \\
& \text { and }\left\|Y_{\iota}(t)+Y_{0}(t)\right\|_{2}=0, \text { for } t>T,
\end{aligned}
$$

then the network (1) is called to be anti-synchronized in finite time.

Design the following controller for the network (1):

$$
u_{\iota}(t)=-W_{\iota}\left(Y_{\iota}(t)+Y_{0}(t)\right)-\eta P^{\frac{a-1}{2}} \operatorname{sign}\left(Y_{\iota}(t)+Y_{0}(t)\right)\left|Y_{\iota}(t)+Y_{0}(t)\right|^{a},
$$

where $W_{\iota} \in \mathbb{R}^{n \times n}, 0<a<1, \operatorname{sign}\left(Y_{\iota}(t)+Y_{0}(t)\right)=\operatorname{diag}\left(\operatorname{sign}\left(Y_{\iota 1}(t)+\right.\right.$ $\left.\left.Y_{01}(t)\right), \operatorname{sign}\left(Y_{\iota 2}(t)+Y_{02}(t)\right), \cdots, \operatorname{sign}\left(Y_{\iota n}(t)+Y_{0 n}(t)\right)\right),\left|Y_{\iota}(t)+Y_{0}(t)\right|^{a}=\left(\mid Y_{\iota 1}(t)+\right.$ $\left.\left.Y_{01}(t)\right|^{a},\left|Y_{\iota 2}(t)+Y_{02}(t)\right|^{a}, \cdots,\left|Y_{\iota n}(t)+Y_{0 n}(t)\right|^{a}\right)^{T}, 0<\eta \in \mathbb{R}, 0<P=$ $\operatorname{diag}\left(p_{1}, p_{2}, \cdots, p_{n}\right) \in \mathbb{R}^{n \times n}, P^{\frac{a-1}{2}}=\operatorname{diag}\left(p_{1}^{\frac{a-1}{2}}, p_{2}^{\frac{a-1}{2}}, \cdots, p_{n}^{\frac{a-1}{2}}\right)$. Then, we have

$$
\begin{aligned}
\dot{Y}_{\iota}(t)= & -A Y_{\iota}(t)+D g\left(Y_{\iota}(t)\right)+\sum_{s=1}^{m} \sum_{j=1}^{\kappa} c_{s} G_{\iota j}^{s} \Gamma_{s} Y_{j}(t)-W_{\iota}\left(Y_{\iota}(t)+Y_{0}(t)\right) \\
& -\eta P^{\frac{a-1}{2}} \operatorname{sign}\left(Y_{\iota}(t)+Y_{0}(t)\right)\left|Y_{\iota}(t)+Y_{0}(t)\right|^{a} .
\end{aligned}
$$

Take $e_{\iota}(t)=Y_{\iota}(t)+Y_{0}(t)$. Then, one can get

$$
\begin{aligned}
\dot{e}_{\iota}(t)= & -A e_{\iota}(t)+D g\left(Y_{\iota}(t)\right)+D g\left(Y_{0}(t)\right)+\sum_{s=1}^{m} \sum_{j=1}^{\kappa} c_{s} G_{\iota j}^{s} \Gamma_{s} e_{j}(t) \\
& -W_{\iota} e_{\iota}(t)-\eta P^{\frac{a-1}{2}} \operatorname{sign}\left(e_{\iota}(t)\right)\left|e_{\iota}(t)\right|^{a} .
\end{aligned}
$$

Theorem 1 If there exist matrices $0<P=\operatorname{diag}\left(p_{1}, p_{2}, \cdots, p_{n}\right) \in \mathbb{R}^{n \times n}$ and $W=\operatorname{diag}\left(W_{1}, W_{2}, \cdots, W_{\kappa}\right) \in \mathbb{R}^{n \kappa \times n \kappa}$ such that

$$
K_{1}+K_{2}+\sum_{s=1}^{m} c_{s} G^{s} \otimes\left(P \Gamma_{s}+\Gamma_{s}^{T} P\right) \leqslant 0,
$$

where $K_{1}=I_{\kappa} \otimes\left(-P A-A P+P D D^{T} P+\Theta\right), K_{2}=-\left(I_{\kappa} \otimes P\right) W-W^{T}\left(I_{\kappa} \otimes\right.$ $P)$, then the network (1) is said to be finite-timely anti-synchronized under the controller (3). What's more, the settling time of anti-synchronization $T$ satisfies $T \leqslant T_{1}=\frac{\left(V_{1}(0)\right)^{\frac{1-a}{2}}}{\eta(1-a)}$. 
Proof. Define the following Lyapunov functional for network (4):

$$
V_{1}(t)=\sum_{\iota=1}^{\kappa} e_{\iota}^{T}(t) P e_{\iota}(t)
$$

Then, one has

$$
\begin{aligned}
\dot{V}_{1}(t)= & 2 \sum_{\iota=1}^{\kappa} e_{\iota}^{T}(t) P\left(-A e_{\iota}(t)+D g\left(Y_{\iota}(t)\right)+\sum_{s=1}^{m} \sum_{j=1}^{\kappa} c_{s} G_{\iota j}^{s} \Gamma_{s} e_{j}(t)\right. \\
& \left.+D g\left(Y_{0}(t)\right)-W_{\iota} e_{\iota}(t)-\eta P^{\frac{a-1}{2}} \operatorname{sign}\left(e_{\iota}(t)\right)\left|e_{\iota}(t)\right|^{a}\right) .
\end{aligned}
$$

Obviously,

$$
2 e_{\iota}^{T}(t) P D\left(g\left(Y_{\iota}(t)\right)+g\left(Y_{0}(t)\right)\right) \leqslant e_{\iota}^{T}(t)\left(P D D^{T} P+\Theta\right) e_{\iota}(t) .
$$

From (7), we can obtain

$$
\begin{aligned}
\dot{V}_{1}(t) \leqslant & 2 \sum_{s=1}^{m} \sum_{\iota=1}^{\kappa} \sum_{j=1}^{\kappa} c_{s} G_{\iota j}^{s} e_{\iota}^{T}(t) P \Gamma_{s} e_{j}(t)+\sum_{\iota=1}^{\kappa} e_{\iota}^{T}(t)\left(-P A-A P+P D D^{T} P\right. \\
& +\Theta) e_{\iota}(t)-2 \sum_{\iota=1}^{\kappa} e_{\iota}^{T}(t) P W_{\iota} e_{\iota}(t)-2 \eta \sum_{\iota=1}^{\kappa} e_{\iota}^{T}(t) P^{\frac{a+1}{2}} \operatorname{sign}\left(e_{\iota}(t)\right)\left|e_{\iota}(t)\right|^{a} \\
= & e^{T}(t)\left[I_{\kappa} \otimes\left(-P A-A P+P D D^{T} P+\Theta\right)+\sum_{s=1}^{m} c_{s} G^{s} \otimes\left(P \Gamma_{s}\right.\right. \\
& \left.\left.+\Gamma_{s}^{T} P\right)-\left(I_{\kappa} \otimes P\right) W-W^{T}\left(I_{\kappa} \otimes P\right)\right] e(t) \\
& -2 \eta \sum_{\iota=1}^{\kappa} e_{\iota}^{T}(t) P^{\frac{a+1}{2}} \operatorname{sign}\left(e_{\iota}(t)\right)\left|e_{\iota}(t)\right|^{a},
\end{aligned}
$$

where $e(t)=\left(e_{1}^{T}(t), e_{2}^{T}(t), \cdots, e_{\kappa}^{T}(t)\right)^{T}$. According to Lemma 2, we can easily obtain

$$
\begin{aligned}
\sum_{\iota=1}^{\kappa} e_{\iota}^{T}(t) P^{\frac{a+1}{2}} \operatorname{sign}\left(e_{\iota}(t)\right)\left|e_{\iota}(t)\right|^{a} & =\sum_{\iota=1}^{\kappa} \sum_{j=1}^{n} p_{j}^{\frac{a+1}{2}}\left|e_{\iota j}(t)\right|^{a+1} \\
& \geqslant \sum_{\iota=1}^{\kappa}\left(\sum_{j=1}^{n} p_{j} e_{\iota j}^{2}(t)\right)^{\frac{a+1}{2}} \\
& =\sum_{\iota=1}^{\kappa}\left(e_{\iota}^{T}(t) P e_{\iota}(t)\right)^{\frac{a+1}{2}} .
\end{aligned}
$$


From (5), (8), (9) and Lemma 2, we can get

$$
\begin{aligned}
\dot{V}_{1}(t) \leqslant & e^{T}(t)\left[I_{\kappa} \otimes\left(-P A-A P+P D D^{T} P+\Theta\right)+\sum_{s=1}^{m} c_{s} G^{s} \otimes\left(P \Gamma_{s}+\Gamma_{s}^{T} P\right)\right. \\
& \left.-\left(I_{\kappa} \otimes P\right) W-W^{T}\left(I_{\kappa} \otimes P\right)\right] e(t)-2 \eta \sum_{\iota=1}^{\kappa}\left(e_{\iota}^{T}(t) P e_{\iota}(t)\right)^{\frac{a+1}{2}} \\
\leqslant & -2 \eta\left(\sum_{\iota=1}^{\kappa} e_{\iota}^{T}(t) P e_{\iota}(t)\right)^{\frac{a+1}{2}} \\
= & -2 \eta\left(V_{1}(t)\right)^{\frac{a+1}{2}} .
\end{aligned}
$$

By Lemma 1, we can get $V_{1}(t)=0, t>T_{1}$ with $T_{1}=\frac{\left(V_{1}(0) \frac{1-a}{2}\right.}{\eta(1-a)}$.

On the other hand,

$$
0 \leqslant \lambda_{m}(P) \sum_{\iota=1}^{\kappa} e_{\iota}^{T}(t) e_{\iota}(t) \leqslant V_{1}(t)
$$

where $\lambda_{m}(P)>0$.

From (10), we obtain $\left\|e_{\iota}(t)\right\|_{2}=0, t>T_{1}$, where $\iota=1,2, \cdots, \kappa$. Therefore, the network (1) achieves finite-time anti-synchronization under the controller (3). The proof is completed.

\subsubsection{Anti-synchronization in finite time for MWCNNs with switching} topology

In this section, we consider the following MWCNNs with switching topology:

$$
\begin{aligned}
\dot{Y}_{\iota}(t)= & -A Y_{\iota}(t)+D g\left(Y_{\iota}(t)\right)+c_{1} \sum_{j=1}^{\kappa} G_{\iota j}^{1, \omega(t)} \Gamma_{1} Y_{j}(t)+c_{2} \sum_{j=1}^{\kappa} G_{\iota j}^{2, \omega(t)} \Gamma_{2} Y_{j}(t)+\cdots \\
& +c_{m} \sum_{j=1}^{\kappa} G_{\iota j}^{m, \omega(t)} \Gamma_{m} Y_{j}(t)+u_{\iota}(t), \quad \iota=1,2, \cdots, \kappa,
\end{aligned}
$$

where $Y_{\iota}(t), g(\cdot), A, D, u_{\iota}(t), c_{s}, \Gamma_{s}(s=1,2, \cdots, m)$ are defined similarly as those in subsection 3.1.1, $\omega(t):[0, \infty) \rightarrow I=\{1,2, \cdots, i\}$ symbols switching signal. For each $\varsigma \in I, G^{s, \varsigma}=\left(G_{\iota j}^{s, \varsigma}\right)_{\kappa \times \kappa}$ represents the coupling configuration matrix in the $s$ th coupling form for the $\varsigma$ th topology, which satisfies $G_{\iota j}^{s, \varsigma}=$ $G_{j \iota}^{s, \varsigma}>0$ if nodes $\iota$ and $j$ are connected for the $\varsigma$ th topology; otherwise, $G_{\iota j}^{s, \varsigma}=G_{j \iota}^{s, \varsigma}=0(\iota \neq j) ;$ and

$$
G_{\iota \iota}^{s, \varsigma}=-\sum_{\substack{j=1 \\ j \neq \iota}}^{\kappa} G_{\iota j}^{s, \varsigma}, \iota=1,2, \cdots, \kappa
$$


For the network (11), design the same controller as (3). Then, we can obtain

$$
\begin{aligned}
\dot{Y}_{\iota}(t)= & -A Y_{\iota}(t)+D g\left(Y_{\iota}(t)\right)+\sum_{s=1}^{m} \sum_{j=1}^{\kappa} c_{s} G_{\iota j}^{s, \varsigma} \Gamma_{s} Y_{j}(t)-W_{\iota}\left(Y_{\iota}(t)+Y_{0}(t)\right) \\
& -\eta P^{\frac{a-1}{2}} \operatorname{sign}\left(Y_{\iota}(t)+Y_{0}(t)\right)\left|Y_{\iota}(t)+Y_{0}(t)\right|^{a}
\end{aligned}
$$

Let $e_{\iota}(t)=Y_{\iota}(t)+Y_{0}(t)$, then

$$
\begin{aligned}
\dot{e}_{\iota}(t)= & -A e_{\iota}(t)+D g\left(Y_{\iota}(t)\right)+D g\left(Y_{0}(t)\right)+\sum_{s=1}^{m} \sum_{j=1}^{\kappa} c_{s} G_{\iota j}^{s, \varsigma} \Gamma_{s} e_{j}(t) \\
& -W_{\iota} e_{\iota}(t)-\eta P^{\frac{a-1}{2}} \operatorname{sign}\left(e_{\iota}(t)\right)\left|e_{\iota}(t)\right|^{a} .
\end{aligned}
$$

Theorem 2 If there exist matrices $0<P=\operatorname{diag}\left(p_{1}, p_{2}, \cdots, p_{n}\right) \in \mathbb{R}^{n \times n}$ and $W=\operatorname{diag}\left(W_{1}, W_{2}, \cdots, W_{\kappa}\right) \in \mathbb{R}^{n \kappa \times n \kappa}$ such that

$$
K_{1}+K_{2}+\sum_{s=1}^{m} c_{s} G^{s, \varsigma} \otimes\left(P \Gamma_{s}+\Gamma_{s}^{T} P\right) \leqslant 0
$$

where $\varsigma=1,2, \cdots, i, K_{1}=I_{\kappa} \otimes\left(-P A-A P+P D D^{T} P+\Theta\right)$ and $K_{2}=$ $-\left(I_{\kappa} \otimes P\right) W-W^{T}\left(I_{\kappa} \otimes P\right)$, then the network (11) is finite-timely anti-synchronized under the controller (3). Moreover, the settling time of anti-synchronization $T$ satisfies $T \leqslant T_{1}=\frac{\left(V_{1}(0)\right)^{\frac{1-a}{2}}}{\eta(1-a)}$.

Proof. Select the same Lyapunov functional as (6) for the network (12). Then, we have

$$
\begin{aligned}
D^{+} V_{1}(t)= & 2 \sum_{\iota=1}^{\kappa} e_{\iota}^{T}(t) P\left(-A e_{\iota}(t)+D g\left(Y_{\iota}(t)\right)+\sum_{s=1}^{m} \sum_{j=1}^{\kappa} c_{s} G_{\iota j}^{s, \varsigma} \Gamma_{s} e_{j}(t)+D g\left(Y_{0}(t)\right)\right. \\
& \left.-W_{\iota} e_{\iota}(t)-\eta P^{\frac{a-1}{2}} \operatorname{sign}\left(e_{\iota}(t)\right)\left|e_{\iota}(t)\right|^{a}\right) \\
\leqslant & 2 \sum_{s=1}^{m} \sum_{\iota=1}^{\kappa} \sum_{j=1}^{\kappa} c_{s} G_{\iota j}^{s, \varsigma} e_{\iota}^{T}(t) P \Gamma_{s} e_{j}(t)+\sum_{\iota=1}^{\kappa} e_{\iota}^{T}(t)\left(-P A-A P+P D D^{T} P\right. \\
& +\Theta) e_{\iota}(t)-2 \sum_{\iota=1}^{\kappa} e_{\iota}^{T}(t) P W_{\iota} e_{\iota}(t)-2 \eta \sum_{\iota=1}^{\kappa} e_{\iota}^{T}(t) P^{\frac{a+1}{2}} \operatorname{sign}\left(e_{\iota}(t)\right)\left|e_{\iota}(t)\right|^{a} \\
= & e^{T}(t)\left[I_{\kappa} \otimes\left(-P A-A P+P D D^{T} P+\Theta\right)+\sum_{s=1}^{m} c_{s} G^{s, \varsigma} \otimes\left(P \Gamma_{s}\right.\right. \\
& \left.\left.+\Gamma_{s}^{T} P\right)-\left(I_{\kappa} \otimes P\right) W-W^{T}\left(I_{\kappa} \otimes P\right)\right] e(t) \\
& -2 \eta \sum_{\iota=1}^{\kappa} e_{\iota}^{T}(t) P^{\frac{a+1}{2}} \operatorname{sign}\left(e_{\iota}(t)\right)\left|e_{\iota}(t)\right|^{a} .
\end{aligned}
$$


It follows from (9), (13) and Lemma 2 that

$$
\begin{aligned}
D^{+} V_{1}(t) & \leqslant-2 \eta \sum_{\iota=1}^{\kappa}\left(e_{\iota}^{T}(t) P e_{\iota}(t)\right)^{\frac{a+1}{2}} \\
& \leqslant-2 \eta\left(\sum_{\iota=1}^{\kappa} e_{\iota}^{T}(t) P e_{\iota}(t)\right)^{\frac{a+1}{2}} \\
& =-2 \eta\left(V_{1}(t)\right)^{\frac{a+1}{2}}
\end{aligned}
$$

Similar to the proof of Theorem 1, we can derive $\left\|e_{\iota}(t)\right\|_{2}=0, t>$ $T_{1}$, where $\iota=1,2, \cdots, \kappa$. Thus, the network (11) reaches finite-time antisynchronization under the controller (3). The proof is completed.

\subsection{Robust anti-synchronization in finite time for MWCNNs}

\subsubsection{Robust anti-synchronization in finite time for $M W C N N s$ with fixed topology}

During the modeling process of CNNs, the existence of environmental noise, limitations of equipment and external interference may lead to the parameters varying within bounded ranges in some circumstances. Therefore, we consider a MWCNNs with parameteric uncertainties consisting of $\kappa$ identical nodes in this section which can be described as

$$
\begin{aligned}
\dot{Y}_{\iota}(t)= & -A Y_{\iota}(t)+D g\left(Y_{\iota}(t)\right)+c_{1} \sum_{j=1}^{\kappa} G_{\iota j}^{1} \Gamma_{1} Y_{j}(t)+c_{2} \sum_{j=1}^{\kappa} G_{\iota j}^{2} \Gamma_{2} Y_{j}(t)+\cdots \\
& +c_{m} \sum_{j=1}^{\kappa} G_{\iota j}^{m} \Gamma_{m} Y_{j}(t)+u_{\iota}(t), \quad \iota=1,2, \cdots, \kappa
\end{aligned}
$$

where $Y_{\iota}(t), g(\cdot), c_{s}, G_{\iota j}^{s}, \Gamma_{s}, s=1,2, \cdots, m, u_{\iota}(t)$ have the same meanings as those in subsection 3.1.1, and the parameters $A$ and $D$ change in the following given ranges:

$$
\left\{\begin{aligned}
A_{I}:= & \left\{A=\operatorname{diag}\left(a_{r}\right): A^{-} \leqslant A \leqslant A^{+}, \text {i.e., } 0<a_{r}^{-} \leqslant a_{r} \leqslant a_{r}^{+},\right. \\
& \left.r=1,2, \cdots, n, \forall A \in A_{I}\right\}, \\
D_{I}:= & \left\{D=\left(d_{r j}\right)_{n \times n}: D^{-} \leqslant D \leqslant D^{+}, i . e ., d_{r j}^{-} \leqslant d_{r j} \leqslant d_{r j}^{+}, r,\right. \\
& \left.j=1,2, \cdots, n, \forall D \in D_{I}\right\} .
\end{aligned}\right.
$$

For convenience, we define

$$
\tilde{d}_{r j}=\max \left\{\left|d_{r j}^{-}\right|,\left|d_{r j}^{+}\right|\right\}, r=1,2, \cdots, n, j=1,2, \cdots, n .
$$


Definition 2 For all $\iota=1,2, \cdots, \kappa, A \in A_{I}$ and $D \in D_{I}$, if there exists a constant $T>0$ satisifying

$$
\begin{aligned}
& \lim _{t \rightarrow T}\left\|Y_{\iota}(t)+Y_{0}(t)\right\|_{2}=0, \\
& \text { and }\left\|Y_{\iota}(t)+Y_{0}(t)\right\|_{2}=0, \text { for } t>T,
\end{aligned}
$$

then the network (14) with the parameter ranges defined by (15) is said to be robustly anti-synchronized in finite time.

For the network (14), design the same controller as (3). Then, we can obtain

$$
\begin{aligned}
\dot{Y}_{\iota}(t)= & -A Y_{\iota}(t)+D g\left(Y_{\iota}(t)\right)+\sum_{s=1}^{m} \sum_{j=1}^{\kappa} c_{s} G_{\iota j}^{s} \Gamma_{s} Y_{j}(t)-W_{\iota}\left(Y_{\iota}(t)+Y_{0}(t)\right) \\
& -\eta P^{\frac{a-1}{2}} \operatorname{sign}\left(Y_{\iota}(t)+Y_{0}(t)\right)\left|Y_{\iota}(t)+Y_{0}(t)\right|^{a} .
\end{aligned}
$$

Let $e_{\iota}(t)=Y_{\iota}(t)+Y_{0}(t)$, then

$$
\begin{aligned}
\dot{e}_{\iota}(t)= & -A e_{\iota}(t)+D g\left(Y_{\iota}(t)\right)+D g\left(Y_{0}(t)\right)+\sum_{s=1}^{m} \sum_{j=1}^{\kappa} c_{s} G_{\iota j}^{s} \Gamma_{s} e_{j}(t) \\
& -W_{\iota} e_{\iota}(t)-\eta P^{\frac{a-1}{2}} \operatorname{sign}\left(e_{\iota}(t)\right)\left|e_{\iota}(t)\right|^{a}
\end{aligned}
$$

where $A$ and $D$ belong to the parameter ranges defined by (15).

Theorem 3 If there exist matrices $0<P=\operatorname{diag}\left(p_{1}, p_{2}, \cdots, p_{n}\right) \in \mathbb{R}^{n \times n}$ and $W=\operatorname{diag}\left(W_{1}, W_{2}, \cdots, W_{\kappa}\right) \in \mathbb{R}^{n \kappa \times n \kappa}$ such that

$$
K_{3}+K_{2}+\sum_{s=1}^{m} c_{s} G^{s} \otimes\left(P \Gamma_{s}+\Gamma_{s}^{T} P\right) \leqslant 0
$$

where $K_{2}=-\left(I_{\kappa} \otimes P\right) W-W^{T}\left(I_{\kappa} \otimes P\right), K_{3}=I_{\kappa} \otimes\left(-P A^{-}-A^{-} P+\varrho_{D} P^{2}+\Theta\right)$, $\varrho_{D}=\sum_{r=1}^{n} \sum_{j=1}^{n} \tilde{d}_{r j}^{2}$, then the network (14) with the parameter ranges defined by (15) is robust finite-timely anti-synchronized under the controller (3). What's more, the settling time of anti-synchronization $T$ satisfies $T \leqslant T_{1}=$ $\frac{\left(V_{1}(0)\right)^{\frac{1-a}{2}}}{\eta(1-a)}$.

Proof. Define the same Lyapunov functional as (6) for network (16). Then, one has

$$
\begin{aligned}
\dot{V}_{1}(t)= & 2 \sum_{\iota=1}^{\kappa} e_{\iota}^{T}(t) P\left(-A e_{\iota}(t)+D g\left(Y_{\iota}(t)\right)+\sum_{s=1}^{m} \sum_{j=1}^{\kappa} c_{s} G_{\iota j}^{s} \Gamma_{s} e_{j}(t)+D g\left(Y_{0}(t)\right)\right. \\
& \left.-W_{\iota} e_{\iota}(t)-\eta P^{\frac{a-1}{2}} \operatorname{sign}\left(e_{\iota}(t)\right)\left|e_{\iota}(t)\right|^{a}\right) .
\end{aligned}
$$


Obviously,

$$
\begin{aligned}
2 \sum_{\iota=1}^{\kappa} e_{\iota}^{T}(t) P D\left(g\left(Y_{\iota}(t)\right)+g\left(Y_{0}(t)\right)\right) & \leqslant \sum_{\iota=1}^{\kappa} e_{\iota}^{T}(t)\left(P D D^{T} P+\Theta\right) e_{\iota}(t) \\
& \leqslant \sum_{\iota=1}^{\kappa} e_{\iota}^{T}(t)\left(\varrho_{D} P^{2}+\Theta\right) e_{\iota}(t) .
\end{aligned}
$$

From (18), we can obtain

$$
\begin{aligned}
\dot{V}_{1}(t) \leqslant & 2 \sum_{s=1}^{m} \sum_{\iota=1}^{\kappa} \sum_{j=1}^{\kappa} c_{s} G_{\iota j}^{s} e_{\iota}^{T}(t) P \Gamma_{s} e_{j}(t)+\sum_{\iota=1}^{\kappa} e_{\iota}^{T}(t)\left(-P A^{-}-A^{-} P\right) e_{\iota}(t) \\
& -2 \sum_{\iota=1}^{\kappa} e_{\iota}^{T}(t) P W_{\iota} e_{\iota}(t)-2 \eta \sum_{\iota=1}^{\kappa} e_{\iota}^{T}(t) P^{\frac{a+1}{2}} \operatorname{sign}\left(e_{\iota}(t)\right)\left|e_{\iota}(t)\right|^{a} \\
& +\sum_{\iota=1}^{\kappa} e_{\iota}^{T}(t)\left(\varrho_{D} P^{2}+\Theta\right) e_{\iota}(t) \\
= & e^{T}(t)\left[I_{\kappa} \otimes\left(-P A^{-}-A^{-} P+\varrho_{D} P^{2}+\Theta\right)+\sum_{s=1}^{m} c_{s} G^{s} \otimes\left(P \Gamma_{s}\right.\right. \\
& \left.\left.+\Gamma_{s}^{T} P\right)-\left(I_{\kappa} \otimes P\right) W-W^{T}\left(I_{\kappa} \otimes P\right)\right] e(t) \\
& -2 \eta \sum_{\iota=1}^{\kappa} e_{\iota}^{T}(t) P^{\frac{a+1}{2}} \operatorname{sign}\left(e_{\iota}(t)\right)\left|e_{\iota}(t)\right|^{a} .
\end{aligned}
$$

It follows from (9), (17) and Lemma 2 that

$$
\begin{aligned}
\dot{V}_{1}(t) & \leqslant-2 \eta \sum_{\iota=1}^{\kappa}\left(e_{\iota}^{T}(t) P e_{\iota}(t)\right)^{\frac{a+1}{2}} \\
& \leqslant-2 \eta\left(\sum_{\iota=1}^{\kappa} e_{\iota}^{T}(t) P e_{\iota}(t)\right)^{\frac{a+1}{2}} \\
& =-2 \eta\left(V_{1}(t)\right)^{\frac{a+1}{2}} .
\end{aligned}
$$

Similar to the proof of Theorem 1 , we can derive $\left\|e_{\iota}(t)\right\|_{2}=0, t>T_{1}$, where $\iota=1,2, \cdots, \kappa$. Hence, the network (14) with the parameter ranges defined by (15) achieves robust finite-time anti-synchronization under the controller (3). The proof is completed. 
3.2.2 Robust anti-synchronization in finite time for MWCNNs with switching topology

In this subsection, we consider the switched MWCNNs with parameter uncertainties:

$$
\begin{aligned}
\dot{Y}_{\iota}(t)= & -A Y_{\iota}(t)+D g\left(Y_{\iota}(t)\right)+c_{1} \sum_{j=1}^{\kappa} G_{\iota j}^{1, \varsigma} \Gamma_{1} Y_{j}(t)+c_{2} \sum_{j=1}^{\kappa} G_{\iota j}^{2, \varsigma} \Gamma_{2} Y_{j}(t)+\cdots \\
& +c_{m} \sum_{j=1}^{\kappa} G_{\iota j}^{m, \varsigma} \Gamma_{m} Y_{j}(t)+u_{\iota}(t), \quad \iota=1,2, \cdots, \kappa,
\end{aligned}
$$

where $Y_{\iota}(t), g(\cdot), u_{\iota}(t), c_{s}, \Gamma_{s}(s=1,2, \cdots, m)$ are defined similarly as those in subsection 3.1.1, $G_{\iota j}^{s, \varsigma}$ has the same definition as in subsection 3.1.2, and $A, D$ belong to the parameter ranges given by (15).

For the network (20), design the same controller as (3). Then, we can obtain

$$
\begin{aligned}
\dot{Y}_{\iota}(t)= & -A Y_{\iota}(t)+D g\left(Y_{\iota}(t)\right)+\sum_{s=1}^{m} \sum_{j=1}^{\kappa} c_{s} G_{\iota j}^{s, \varsigma} \Gamma_{s} Y_{j}(t)-W_{\iota}\left(Y_{\iota}(t)+Y_{0}(t)\right) \\
& -\eta P^{\frac{a-1}{2}} \operatorname{sign}\left(Y_{\iota}(t)+Y_{0}(t)\right)\left|Y_{\iota}(t)+Y_{0}(t)\right|^{a}
\end{aligned}
$$

Let $e_{\iota}(t)=Y_{\iota}(t)+Y_{0}(t)$, then

$$
\begin{aligned}
\dot{e}_{\iota}(t)= & -A e_{\iota}(t)+D g\left(Y_{\iota}(t)\right)+D g\left(Y_{0}(t)\right)+\sum_{s=1}^{m} \sum_{j=1}^{\kappa} c_{s} G_{\iota j}^{s, \varsigma} \Gamma_{s} e_{j}(t) \\
& -W_{\iota} e_{\iota}(t)-\eta P^{\frac{a-1}{2}} \operatorname{sign}\left(e_{\iota}(t)\right)\left|e_{\iota}(t)\right|^{a},
\end{aligned}
$$

in which the parameters $A$ and $D$ change in some given parameter ranges defined by (15).

Theorem 4 If there exist matrices $0<P=\operatorname{diag}\left(p_{1}, p_{2}, \cdots, p_{n}\right) \in \mathbb{R}^{n \times n}$ and $W=\operatorname{diag}\left(W_{1}, W_{2}, \cdots, W_{\kappa}\right) \in \mathbb{R}^{n \kappa \times n \kappa}$ such that

$$
K_{3}+K_{2}+\sum_{s=1}^{m} c_{s} G^{s, \varsigma} \otimes\left(P \Gamma_{s}+\Gamma_{s}^{T} P\right) \leqslant 0
$$

where $\varsigma=1,2, \cdots, i, K_{3}=I_{\kappa} \otimes\left(-P A^{-}-A^{-} P+\varrho_{D} P^{2}+\Theta\right), K_{2}=-\left(I_{\kappa} \otimes\right.$ $P) W-W^{T}\left(I_{\kappa} \otimes P\right), \varrho_{D}=\sum_{r=1}^{n} \sum_{j=1}^{n} \tilde{d}_{r j}^{2}$, then the network (20) with the parameter ranges defined by (15) is robust finite-timely anti-synchronized under the controller (3). Moreover, the settling time of anti-synchronization $T$ satisfies $T \leqslant T_{1}=\frac{\left(V_{1}(0)\right)^{\frac{1-a}{2}}}{\eta(1-a)}$. 
Proof. Select the same Lyapunov functional as (6) for the network (21). Then, one has

$$
\begin{aligned}
D^{+} V_{1}(t)= & 2 \sum_{\iota=1}^{\kappa} e_{\iota}^{T}(t) P\left(-A e_{\iota}(t)+D g\left(Y_{\iota}(t)\right)+\sum_{s=1}^{m} \sum_{j=1}^{\kappa} c_{s} G_{\iota j}^{s, \varsigma} \Gamma_{s} e_{j}(t)+D g\left(Y_{0}(t)\right)\right. \\
& \left.-W_{\iota} e_{\iota}(t)-\eta P^{\frac{a-1}{2}} \operatorname{sign}\left(e_{\iota}(t)\right)\left|e_{\iota}(t)\right|^{a}\right) \\
\leqslant & 2 \sum_{s=1}^{m} \sum_{\iota=1}^{\kappa} \sum_{j=1}^{\kappa} c_{s} G_{\iota \iota}^{s, \varsigma} e_{\iota}^{T}(t) P \Gamma_{s} e_{j}(t)+\sum_{\iota=1}^{\kappa} e_{\iota}^{T}(t)\left(-P A^{-}-A^{-} P\right) e_{\iota}(t) \\
& -2 \sum_{\iota=1}^{\kappa} e_{\iota}^{T}(t) P W_{\iota} e_{\iota}(t)-2 \eta \sum_{\iota=1}^{\kappa} e_{\iota}^{T}(t) P^{\frac{a+1}{2}} \operatorname{sign}\left(e_{\iota}(t)\right)\left|e_{\iota}(t)\right|^{a} \\
& +\sum_{\iota=1}^{\kappa} e_{\iota}^{T}(t)\left(\varrho_{D} P^{2}+\Theta\right) e_{\iota}(t) \\
= & e^{T}(t)\left[I_{\kappa} \otimes\left(-P A^{-}-A^{-} P+\varrho_{D} P^{2}+\Theta\right)+\sum_{s=1}^{m} c_{s} G^{s, \varsigma} \otimes\left(P \Gamma_{s}\right.\right. \\
& \left.\left.+\Gamma_{s}^{T} P\right)-\left(I_{\kappa} \otimes P\right) W-W^{T}\left(I_{\kappa} \otimes P\right)\right] e(t) \\
& -2 \eta \sum_{\iota=1}^{\kappa} e_{\iota}^{T}(t) P^{\frac{a+1}{2}} \operatorname{sign}\left(e_{\iota}(t)\right)\left|e_{\iota}(t)\right|^{a} .
\end{aligned}
$$

It follows from (9), (22) and Lemma 2 that

$$
\begin{aligned}
D^{+} V_{1}(t) & \leqslant-2 \eta \sum_{\iota=1}^{\kappa}\left(e_{\iota}^{T}(t) P e_{\iota}(t)\right)^{\frac{a+1}{2}} \\
& \leqslant-2 \eta\left(\sum_{\iota=1}^{\kappa} e_{\iota}^{T}(t) P e_{\iota}(t)\right)^{\frac{a+1}{2}} \\
& =-2 \eta\left(V_{1}(t)\right)^{\frac{a+1}{2}} .
\end{aligned}
$$

Similar to the proof of Theorem 1, we can derive $\left\|e_{\iota}(t)\right\|_{2}=0, t>T_{1}$, where $\iota=1,2, \cdots, \kappa$. Therefore, the network (20) with the parameter ranges defined by (15) achieves robust finite-time anti-synchronization under the controller (3). The proof is completed.

Remark 1 In recent years, some meaningful research results have been reported on the dynamical behaviors of Markovian jump systems [55-61]. Shen et al. [56] investigated the finite-time event-triggered $H_{\infty}$ control problem for Takagi-Sugeno Markov jump fuzzy systems. As is well known, Markov jump systems can be used to model some real-life systems experiencing random changes in their parameters or structures. Therefore, it would be very interesting to apply a Markov process into the MWCNNs considered in our paper and investigate the finite-time anti-synchronization of MWCNNs with Markovian jump topology. This would be a meaningful problem for our future research. 


\section{Finite-time anti-synchronization of MWCNNs with coupling} delays

4.1 Anti-synchronization in finite time for delayed MWCNNs

\subsubsection{Anti-synchronization in finite time for delayed MWCNNs with fixed} topology

In this section, we consider the following network model of MWCNNs with coupling delays:

$$
\dot{Y}_{\iota}(t)=-A Y_{\iota}(t)+D g\left(Y_{\iota}(t)\right)+\sum_{s=1}^{m} \sum_{j=1}^{\kappa} c_{s} G_{\iota j}^{s} \Gamma_{s} Y_{j}\left(t-\tau_{s}(t)\right)+u_{\iota}(t)
$$

where $\iota=1,2, \cdots, \kappa, Y_{\iota}(t), g(\cdot), A, D, c_{s}, G_{\iota j}^{s}, \Gamma_{s}, u_{\iota}(t)$ are defined similarly as those in subsection 3.1.1, $\tau_{s}(t)$ is the time-varying delay with $0 \leqslant \tau_{s}(t) \leqslant \tau$, $s=1,2, \cdots, m$.

Construct the controller for the network (23) as follows:

$$
\begin{aligned}
u_{\iota}(t)= & -\eta P^{-1} \sum_{s=1}^{m}\left(\frac{c_{s}}{1-\gamma_{s}} \int_{t-\tau_{s}(t)}^{t}\left(Y_{\iota}(h)+Y_{0}(h)\right)^{T} Q_{\iota}^{s}\left(Y_{\iota}(h)+Y_{0}(h)\right) d h\right)^{\frac{a+1}{2}} \\
& \times \frac{Y_{\iota}(t)+Y_{0}(t)}{\left\|Y_{\iota}(t)+Y_{0}(t)\right\|_{2}^{2}}-\eta P^{\frac{a-1}{2}} \operatorname{sign}\left(Y_{\iota}(t)+Y_{0}(t)\right)\left|Y_{\iota}(t)+Y_{0}(t)\right|^{a} \\
& -W_{\iota}\left(Y_{\iota}(t)+Y_{0}(t)\right) .
\end{aligned}
$$

where $0<Q_{\iota}^{s} \in \mathbb{R}^{n \times n}, Q_{s}=\operatorname{diag}\left(Q_{1}^{s}, Q_{2}^{s}, \cdots, Q_{\kappa}^{s}\right), s=1,2, \cdots, m, W_{\iota}, a, P$, $\eta, \operatorname{sign}\left(Y_{\iota}(t)+Y_{0}(t)\right),\left|Y_{\iota}(t)+Y_{0}(t)\right|^{a}$ have the same meanings as in (3). Then, we have

$$
\begin{aligned}
\dot{Y}_{\iota}(t)= & -A Y_{\iota}(t)+D g\left(Y_{\iota}(t)\right)+\sum_{s=1}^{m} \sum_{j=1}^{\kappa} c_{s} G_{\iota j}^{s} \Gamma_{s} Y_{j}\left(t-\tau_{s}(t)\right)-W_{\iota}\left(Y_{\iota}(t)+Y_{0}(t)\right) \\
& -\eta P^{-1} \sum_{s=1}^{m}\left(\frac{c_{s}}{1-\gamma_{s}} \int_{t-\tau_{s}(t)}^{t}\left(Y_{\iota}(h)+Y_{0}(h)\right)^{T} Q_{\iota}^{s}\left(Y_{\iota}(h)+Y_{0}(h)\right) d h\right)^{\frac{a+1}{2}} \\
& \times \frac{Y_{\iota}(t)+Y_{0}(t)}{\left\|Y_{\iota}(t)+Y_{0}(t)\right\|_{2}^{2}}-\eta P^{\frac{a-1}{2}} \operatorname{sign}\left(Y_{\iota}(t)+Y_{0}(t)\right)\left|Y_{\iota}(t)+Y_{0}(t)\right|^{a} .
\end{aligned}
$$

Let $e_{\iota}(t)=Y_{\iota}(t)+Y_{0}(t)$. Obviously, we can obtain

$$
\begin{aligned}
\dot{e}_{\iota}(t)= & -A e_{\iota}(t)+D g\left(Y_{\iota}(t)\right)+D g\left(Y_{0}(t)\right)+\sum_{s=1}^{m} \sum_{j=1}^{\kappa} c_{s} G_{\iota j}^{s} \Gamma_{s} e_{j}\left(t-\tau_{s}(t)\right)-W_{\iota} e_{\iota}(t) \\
& -\eta P^{-1} \sum_{s=1}^{m}\left(\frac{c_{s}}{1-\gamma_{s}} \int_{t-\tau_{s}(t)}^{t} e_{\iota}^{T}(h) Q_{\iota}^{s} e_{\iota}(h) d h\right)^{\frac{a+1}{2}} \frac{e_{\iota}(t)}{\left\|e_{\iota}(t)\right\|_{2}^{2}} \\
& -\eta P^{\frac{a-1}{2}} \operatorname{sign}\left(e_{\iota}(t)\right)\left|e_{\iota}(t)\right|^{a}
\end{aligned}
$$


where $\iota=1,2, \cdots, \kappa$.

Theorem 5 Suppose $\dot{\tau}_{s}(t) \leqslant \gamma_{s}<1$. The network (23) realizes finite-time anti-synchronization under the controller (24) if there exist matrices $0<P=$ $\operatorname{diag}\left(p_{1}, p_{2}, \cdots, p_{n}\right) \in \mathbb{R}^{n \times n}, W=\operatorname{diag}\left(W_{1}, W_{2}, \cdots, W_{\kappa}\right) \in \mathbb{R}^{n \kappa \times n \kappa}$ and $0<$ $Q_{s}=\operatorname{diag}\left(Q_{1}^{s}, Q_{2}^{s}, \cdots, Q_{\kappa}^{s}\right) \in \mathbb{R}^{n \kappa \times n \kappa}$, such that

$$
K_{1}+K_{2}+\sum_{s=1}^{m} c_{s}\left(F_{s}^{T} Q_{s}^{-1} F_{s}+\frac{1}{1-\gamma_{s}} Q_{s}\right) \leqslant 0
$$

where $K_{1}=I_{\kappa} \otimes\left(-P A-A P+P D D^{T} P+\Theta\right), K_{2}=-\left(I_{\kappa} \otimes P\right) W-W^{T}\left(I_{\kappa} \otimes\right.$ $P), F_{s}=G^{s} \otimes\left(\Gamma_{s}^{T} P\right)$. In addition, the settling time of anti-synchronization $T$ satisfies $T \leqslant T_{2}=\frac{\left(V_{2}(0) \frac{1-a}{2}\right.}{\eta(1-a)}$.

Proof. Construct a Lyapunov functional for network (25):

$$
V_{2}(t)=\sum_{\iota=1}^{\kappa} e_{\iota}^{T}(t) P e_{\iota}(t)+\sum_{s=1}^{m} \frac{c_{s}}{1-\gamma_{s}} \int_{t-\tau_{s}(t)}^{t} e^{T}(h) Q_{s} e(h) d h .
$$

Then,

$$
\begin{aligned}
\dot{V}_{2}(t) \leqslant & 2 \sum_{\iota=1}^{\kappa} e_{\iota}^{T}(t) P\left(-A e_{\iota}(t)+D g\left(Y_{\iota}(t)\right)+D g\left(Y_{0}(t)\right)+\sum_{s=1}^{m} \sum_{j=1}^{\kappa} c_{s} G_{\iota j}^{s} \Gamma_{s} e_{j}\left(t-\tau_{s}(t)\right)\right. \\
& \left.-W_{\iota} e_{\iota}(t)-\eta P^{\frac{a-1}{2}} \operatorname{sign}\left(e_{\iota}(t)\right)\left|e_{\iota}(t)\right|^{a}\right)-\sum_{s=1}^{m} c_{s} e^{T}\left(t-\tau_{s}(t)\right) Q_{s} e\left(t-\tau_{s}(t)\right) \\
& -2 \eta \sum_{\iota=1}^{\kappa} \sum_{s=1}^{m}\left(\frac{c_{s}}{1-\gamma_{s}} \int_{t-\tau_{s}(t)}^{t} e_{\iota}^{T}(h) Q_{\iota}^{s} e_{\iota}(h) d h\right)^{\frac{a+1}{2}}+\sum_{s=1}^{m} \frac{c_{s}}{1-\gamma_{s}} e^{T}(t) Q_{s} e(t)
\end{aligned}
$$

where $e\left(t-\tau_{s}(t)\right)=\left(e_{1}^{T}\left(t-\tau_{s}(t)\right), e_{2}^{T}\left(t-\tau_{s}(t)\right), \cdots, e_{\kappa}^{T}\left(t-\tau_{s}(t)\right)\right)^{T}$. Obviously,

$$
\begin{aligned}
& 2 \sum_{s=1}^{m} \sum_{\iota=1}^{\kappa} \sum_{j=1}^{\kappa} c_{s} G_{\iota j}^{s} e_{\iota}^{T}(t) P \Gamma_{s} e_{j}\left(t-\tau_{s}(t)\right) \\
= & 2 \sum_{s=1}^{m} c_{s} e^{T}(t)\left[G^{s} \otimes\left(P \Gamma_{s}\right)\right] e\left(t-\tau_{s}(t)\right) \\
\leqslant & \left.\sum_{s=1}^{m} c_{s} e^{T}(t)\left[G^{s} \otimes\left(P \Gamma_{s}\right)\right] Q_{s}^{-1}\left[G^{s} \otimes\left(\Gamma_{s}^{T} P\right)\right)\right] e(t) \\
& +\sum_{s=1}^{m} c_{s} e^{T}\left(t-\tau_{s}(t)\right) Q_{s} e\left(t-\tau_{s}(t)\right) .
\end{aligned}
$$


From (7), (9), (26), (28) and Lemma 2, we can obtain

$$
\begin{aligned}
\dot{V}_{2}(t) \leqslant & e^{T}(t)\left[I_{\kappa} \otimes\left(-P A-A P+P D D^{T} P+\Theta\right)\right] e(t)+\sum_{s=1}^{m} \frac{c_{s}}{1-\gamma_{s}} e^{T}(t) Q_{s} e(t) \\
& +\sum_{s=1}^{m} c_{s} e^{T}(t)\left[G^{s} \otimes\left(P \Gamma_{s}\right)\right] Q_{s}^{-1}\left[G^{s} \otimes\left(\Gamma_{s}^{T} P\right)\right] e(t)-2 \sum_{\iota=1}^{\kappa} e_{\iota}^{T}(t) P W_{\iota} e_{\iota}(t) \\
& -2 \eta \sum_{\iota=1}^{\kappa}\left(e_{\iota}^{T}(t) P e_{\iota}(t)\right)^{\frac{a+1}{2}}-2 \eta \sum_{s=1}^{m}\left(\frac{c_{s}}{1-\gamma_{s}} \int_{t-\tau_{s}(t)}^{t} e^{T}(h) Q_{s} e(h) d h\right)^{\frac{a+1}{2}} \\
= & e^{T}(t)\left\{I_{\kappa} \otimes\left(-P A-A P+P D D^{T} P+\Theta\right)+\sum_{s=1}^{m} \frac{c_{s}}{1-\gamma_{s}} Q_{s}-\left(I_{\kappa} \otimes P\right) W\right. \\
& \left.-W^{T}\left(I_{\kappa} \otimes P\right)+\sum_{s=1}^{m} c_{s}\left[G^{s} \otimes\left(P \Gamma_{s}\right)\right] Q_{s}^{-1}\left[G^{s} \otimes\left(\Gamma_{s}^{T} P\right)\right]\right\} e(t) \\
& -2 \eta \sum_{s=1}^{m}\left(\frac{c_{s}}{1-\gamma_{s}} \int_{t-\tau_{s}(t)}^{t} e^{T}(h) Q_{s} e(h) d h\right)^{\frac{a+1}{2}} \\
& -2 \eta \sum_{\iota=1}^{\kappa}\left(e_{\iota}^{T}(t) P e_{\iota}(t)\right)^{\frac{a+1}{2}} \\
\leqslant & -2 \eta \sum_{\iota=1}^{\kappa}\left(e_{\iota}^{T}(t) P e_{\iota}(t)\right)^{\frac{a+1}{2}}-2 \eta \sum_{s=1}^{m}\left(\frac{c_{s}}{1-\gamma_{s}} \int_{t-\tau_{s}(t)}^{t} e^{T}(h) Q_{s} e(h) d h\right)^{\frac{a+1}{2}} \\
\leqslant & -2 \eta\left(\sum_{\iota=1}^{\kappa} e_{\iota}^{T}(t) P e_{\iota}(t)+\sum_{s=1}^{m} \frac{c_{s}}{1-\gamma_{s}} \int_{t-\tau_{s}(t)}^{t} e^{T}(h) Q_{s} e(h) d h\right)^{\frac{a+1}{2}} \\
= & -2 \eta\left(V_{2}(t)\right)^{\frac{a+1}{2}} .
\end{aligned}
$$

Similar to the proof of Theorem 1, it is easy to obtain $\left\|e_{\iota}(t)\right\|_{2}=0, \iota=$ $1,2, \cdots, \kappa, t>T_{2}$. Obviously, the network (23) is finite-time anti-synchronization. The proof is completed.

\subsubsection{Anti-synchronization in finite time for delayed MWCNNs with} switching topology

Describe the network model of MWCNNs with coupling delays and switching topology in this section as follows:

$$
\dot{Y}_{\iota}(t)=-A Y_{\iota}(t)+D g\left(Y_{\iota}(t)\right)+\sum_{s=1}^{m} \sum_{j=1}^{\kappa} c_{s} G_{\iota j}^{s, \varsigma} \Gamma_{s} Y_{j}\left(t-\tau_{s}(t)\right)+u_{\iota}(t)
$$

where $\iota=1,2, \cdots, \kappa, Y_{\iota}(t), g(\cdot), A, D, c_{s}, u_{\iota}(t), \Gamma_{s}$ represent the same definitions as in subsection 3.1.1, $\tau_{s}(t)$ is the time-varying delay as that in subsection 4.1.1, and $G_{\iota j}^{s, \varsigma}$ has the same meaning as that in subsection 3.1.2. 
Construct the same controller as (24) for the network (29). Then, one has

$$
\begin{aligned}
\dot{Y}_{\iota}(t)= & -A Y_{\iota}(t)+D g\left(Y_{\iota}(t)\right)+\sum_{s=1}^{m} \sum_{j=1}^{\kappa} c_{s} G_{\iota j}^{s, \varsigma} \Gamma_{s} Y_{j}\left(t-\tau_{s}(t)\right)-W_{\iota}\left(Y_{\iota}(t)+Y_{0}(t)\right) \\
& -\eta P^{-1} \sum_{s=1}^{m}\left(\frac{c_{s}}{1-\gamma_{s}} \int_{t-\tau_{s}(t)}^{t}\left(Y_{\iota}(h)+Y_{0}(h)\right)^{T} Q_{\iota}^{s}\left(Y_{\iota}(h)+Y_{0}(h)\right) d h\right)^{\frac{a+1}{2}} \\
& \times \frac{Y_{\iota}(t)+Y_{0}(t)}{\left\|Y_{\iota}(t)+Y_{0}(t)\right\|_{2}^{2}}-\eta P^{\frac{a-1}{2}} \operatorname{sign}\left(Y_{\iota}(t)+Y_{0}(t)\right)\left|Y_{\iota}(t)+Y_{0}(t)\right|^{a} .
\end{aligned}
$$

Let $e_{\iota}(t)=Y_{\iota}(t)+Y_{0}(t)$. Then, we have

$$
\begin{aligned}
\dot{e}_{\iota}(t)= & -A e_{\iota}(t)+D g\left(Y_{\iota}(t)\right)+D g\left(Y_{0}(t)\right)+\sum_{s=1}^{m} \sum_{j=1}^{\kappa} c_{s} G_{\iota j}^{s, \varsigma} \Gamma_{s} e_{j}\left(t-\tau_{s}(t)\right)-W_{\iota} e_{\iota}(t) \\
& -\eta P^{-1} \sum_{s=1}^{m}\left(\frac{c_{s}}{1-\gamma_{s}} \int_{t-\tau_{s}(t)}^{t} e_{\iota}^{T}(h) Q_{\iota}^{s} e_{\iota}(h) d h\right)^{\frac{a+1}{2}} \frac{e_{\iota}(t)}{\left\|e_{\iota}(t)\right\|_{2}^{2}} \\
& -\eta P^{\frac{a-1}{2}} \operatorname{sign}\left(e_{\iota}(t)\right)\left|e_{\iota}(t)\right|^{a}
\end{aligned}
$$

where $\iota=1,2, \cdots, \kappa$.

Theorem 6 Suppose $\dot{\tau}_{s}(t) \leqslant \gamma_{s}<1$. The network (29) realizes finite-time anti-synchronization under the controller (24) if there exist matrices $0<P=$ $\operatorname{diag}\left(p_{1}, p_{2}, \cdots, p_{n}\right) \in \mathbb{R}^{n \times n}, W=\operatorname{diag}\left(W_{1}, W_{2}, \cdots, W_{\kappa}\right) \in \mathbb{R}^{n \kappa \times n \kappa}$ and $0<$ $Q_{s}=\operatorname{diag}\left(Q_{1}^{s}, Q_{2}^{s}, \cdots, Q_{\kappa}^{s}\right) \in \mathbb{R}^{n \kappa \times n \kappa}$, such that

$$
K_{1}+K_{2}+\sum_{s=1}^{m} c_{s}\left(F_{s, \varsigma}^{T} Q_{s}^{-1} F_{s, \varsigma}+\frac{1}{1-\gamma_{s}} Q_{s}\right) \leqslant 0
$$

where $\varsigma=1,2, \cdots, i, K_{1}=I_{\kappa} \otimes\left(-P A-A P+P D D^{T} P+\Theta\right), K_{2}=-\left(I_{\kappa} \otimes\right.$ $P) W-W^{T}\left(I_{\kappa} \otimes P\right)$ and $F_{s, \varsigma}=G^{s, \varsigma} \otimes\left(\Gamma_{s}^{T} P\right)$. What's more, the settling time of anti-synchronization $T$ satisfies $T \leqslant T_{2}=\frac{\left(V_{2}(0)\right)^{\frac{1-a}{2}}}{\eta(1-a)}$. 
Proof. Construct the same Lyapunov functional as (24) for network (30). Then,

$$
\begin{aligned}
& D^{+} V_{2}(t) \leqslant 2 \sum_{\iota=1}^{\kappa} e_{\iota}^{T}(t) P\left(-A e_{\iota}(t)+D g\left(Y_{\iota}(t)\right)+D g\left(Y_{0}(t)\right)+\sum_{s=1}^{m} \sum_{j=1}^{\kappa} c_{s} G_{\iota j}^{s, \varsigma} \Gamma_{s} e_{j}(t\right. \\
& \left.\left.-\tau_{s}(t)\right)-W_{\iota} e_{\iota}(t)-\eta P^{\frac{a-1}{2}} \operatorname{sign}\left(e_{\iota}(t)\right)\left|e_{\iota}(t)\right|^{a}\right)+\sum_{s=1}^{m} \frac{c_{s}}{1-\gamma_{s}} e^{T}(t) Q_{s} e(t) \\
& -2 \eta \sum_{\iota=1}^{\kappa} \sum_{s=1}^{m}\left(\frac{c_{s}}{1-\gamma_{s}} \int_{t-\tau_{s}(t)}^{t} e_{\iota}^{T}(h) Q_{\iota}^{s} e_{\iota}(h) d h\right)^{\frac{a+1}{2}}-\sum_{s=1}^{m} c_{s} e^{T}(t \\
& \left.-\tau_{s}(t)\right) Q_{s} e\left(t-\tau_{s}(t)\right) \\
& \leqslant e^{T}(t)\left[I_{\kappa} \otimes\left(-P A-A P+P D D^{T} P+\Theta\right)\right] e(t)+\sum_{s=1}^{m} \frac{c_{s}}{1-\gamma_{s}} e^{T}(t) Q_{s} e(t) \\
& +\sum_{s=1}^{m} c_{s} e^{T}(t)\left[G^{s, \varsigma} \otimes\left(P \Gamma_{s}\right)\right] Q_{s}^{-1}\left[G^{s, \varsigma} \otimes\left(\Gamma_{s}^{T} P\right)\right] e(t)-2 \sum_{\iota=1}^{\kappa} e_{\iota}^{T}(t) P W_{\iota} e_{\iota}(t) \\
& -2 \eta \sum_{\iota=1}^{\kappa}\left(e_{\iota}^{T}(t) P e_{\iota}(t)\right)^{\frac{a+1}{2}}-2 \eta \sum_{s=1}^{m}\left(\frac{c_{s}}{1-\gamma_{s}} \int_{t-\tau_{s}(t)}^{t} e^{T}(h) Q_{s} e(h) d h\right)^{\frac{a+1}{2}} \\
& =e^{T}(t)\left\{I_{\kappa} \otimes\left(-P A-A P+P D D^{T} P+\Theta\right)+\sum_{s=1}^{m} \frac{c_{s}}{1-\gamma_{s}} Q_{s}-\left(I_{\kappa} \otimes P\right) W\right. \\
& \left.-W^{T}\left(I_{\kappa} \otimes P\right)+\sum_{s=1}^{m} c_{s}\left[G^{s, \varsigma} \otimes\left(P \Gamma_{s}\right)\right] Q_{s}^{-1}\left[G^{s, \varsigma} \otimes\left(\Gamma_{s}^{T} P\right)\right]\right\} e(t) \\
& -2 \eta \sum_{s=1}^{m}\left(\frac{c_{s}}{1-\gamma_{s}} \int_{t-\tau_{s}(t)}^{t} e^{T}(h) Q_{s} e(h) d h\right)^{\frac{a+1}{2}} \\
& -2 \eta \sum_{\iota=1}^{\kappa}\left(e_{\iota}^{T}(t) P e_{\iota}(t)\right)^{\frac{a+1}{2}} \\
& \leqslant-2 \eta \sum_{\iota=1}^{\kappa}\left(e_{\iota}^{T}(t) P e_{\iota}(t)\right)^{\frac{a+1}{2}}-2 \eta \sum_{s=1}^{m}\left(\frac{c_{s}}{1-\gamma_{s}} \int_{t-\tau_{s}(t)}^{t} e^{T}(h) Q_{s} e(h) d h\right)^{\frac{a+1}{2}} \\
& \leqslant-2 \eta\left(\sum_{\iota=1}^{\kappa} e_{\iota}^{T}(t) P e_{\iota}(t)+\sum_{s=1}^{m} \frac{c_{s}}{1-\gamma_{s}} \int_{t-\tau_{s}(t)}^{t} e^{T}(h) Q_{s} e(h) d h\right)^{\frac{a+1}{2}} \\
& =-2 \eta\left(V_{2}(t)\right)^{\frac{a+1}{2}} \text {. }
\end{aligned}
$$

Similar to the proof of Theorem 1 , it is easy to obtain $\left\|e_{\iota}(t)\right\|_{2}=0, \iota=$ $1,2, \cdots, \kappa, t>T_{2}$. Obviously, the network (29) achieves finite-time antisynchronization. The proof is completed. 
4.2 Robust anti-synchronization in finite time for delayed MWCNNs

4.2.1 Robust anti-synchronization in finite time for delayed MWCNNs with fixed topology

The MWCNNs model with coupling delays and parameter uncertainties is described by:

$$
\dot{Y}_{\iota}(t)=-A Y_{\iota}(t)+D g\left(Y_{\iota}(t)\right)+\sum_{s=1}^{m} \sum_{j=1}^{\kappa} c_{s} G_{\iota j}^{s} \Gamma_{s} Y_{j}\left(t-\tau_{s}(t)\right)+u_{\iota}(t)
$$

where $\iota=1,2, \cdots, \kappa, Y_{\iota}(t), g(\cdot), c_{s}, G_{\iota j}^{s}, \Gamma_{s}, u_{\iota}(t)$ are defined similarly as those in subsection 3.1.1, $A, D$ are intervalized as those in $(15), \tau_{s}(t)$ is the time-varying delay as that in subsection 4.1.1.

Design the same controller for the network (32) as (24). Then,

$$
\begin{aligned}
\dot{Y}_{\iota}(t)= & -A Y_{\iota}(t)+D g\left(Y_{\iota}(t)\right)+\sum_{s=1}^{m} \sum_{j=1}^{\kappa} c_{s} G_{\iota j}^{s} \Gamma_{s} Y_{j}\left(t-\tau_{s}(t)\right)-W_{\iota}\left(Y_{\iota}(t)+Y_{0}(t)\right) \\
& -\eta P^{-1} \sum_{s=1}^{m}\left(\frac{c_{s}}{1-\gamma_{s}} \int_{t-\tau_{s}(t)}^{t}\left(Y_{\iota}(h)+Y_{0}(h)\right)^{T} Q_{\iota}^{s}\left(Y_{\iota}(h)+Y_{0}(h)\right) d h\right)^{\frac{a+1}{2}} \\
& \times \frac{Y_{\iota}(t)+Y_{0}(t)}{\left\|Y_{\iota}(t)+Y_{0}(t)\right\|_{2}^{2}}-\eta P^{\frac{a-1}{2}} \operatorname{sign}\left(Y_{\iota}(t)+Y_{0}(t)\right)\left|Y_{\iota}(t)+Y_{0}(t)\right|^{a} .
\end{aligned}
$$

Let $e_{\iota}(t)=Y_{\iota}(t)+Y_{0}(t)$. Then, we have

$$
\begin{aligned}
\dot{e}_{\iota}(t)= & -A e_{\iota}(t)+D g\left(Y_{\iota}(t)\right)+D g\left(Y_{0}(t)\right)+\sum_{s=1}^{m} \sum_{j=1}^{\kappa} c_{s} G_{\iota j}^{s} \Gamma_{s} e_{j}\left(t-\tau_{s}(t)\right)-W_{\iota} e_{\iota}(t) \\
& -\eta P^{-1} \sum_{s=1}^{m}\left(\frac{c_{s}}{1-\gamma_{s}} \int_{t-\tau_{s}(t)}^{t} e_{\iota}^{T}(h) Q_{\iota}^{s} e_{\iota}(h) d h\right)^{\frac{a+1}{2}} \frac{e_{\iota}(t)}{\left\|e_{\iota}(t)\right\|_{2}^{2}} \\
& -\eta P^{\frac{a-1}{2}} \operatorname{sign}\left(e_{\iota}(t)\right)\left|e_{\iota}(t)\right|^{a}
\end{aligned}
$$

where $\iota=1,2, \cdots, \kappa, A$ and $D$ are uncertain parameters which belong to the ranges given in (15).

Theorem 7 Suppose $\dot{\tau}_{s}(t) \leqslant \gamma_{s}<1$. The network (32) with the parameter ranges defined by (15) achieves robust finite-time anti-synchronization under the controller (24) if there exist matrices $0<P=\operatorname{diag}\left(p_{1}, p_{2}, \cdots, p_{n}\right) \in \mathbb{R}^{n \times n}$, $W=\operatorname{diag}\left(W_{1}, W_{2}, \cdots, W_{\kappa}\right) \in \mathbb{R}^{n \kappa \times n \kappa}$ and $0<Q_{s}=\operatorname{diag}\left(Q_{1}^{s}, Q_{2}^{s}, \cdots, Q_{\kappa}^{s}\right) \in$ $\mathbb{R}^{n \kappa \times n \kappa}$, such that

$$
K_{3}+K_{2}+\sum_{s=1}^{m} c_{s}\left(F_{s}^{T} Q_{s}^{-1} F_{s}+\frac{1}{1-\gamma_{s}} Q_{s}\right) \leqslant 0
$$


where $K_{3}=I_{\kappa} \otimes\left(-P A^{-}-A^{-} P+\varrho_{D} P^{2}+\Theta\right), \varrho_{D}=\sum_{r=1}^{n} \sum_{j=1}^{n} \tilde{d}_{r j}^{2}, K_{2}=$ $-\left(I_{\kappa} \otimes P\right) W-W^{T}\left(I_{\kappa} \otimes P\right), F_{s}=G^{s} \otimes\left(\Gamma_{s}^{T} P\right)$. In addition, the settling time of anti-synchronization $T$ satisfies $T \leqslant T_{2}=\frac{\left(V_{2}(0)\right)^{\frac{1-a}{2}}}{\eta(1-a)}$.

Proof. The Lyapunov functional is constructed similarly as that in Theorem 5 for network (33). Then,

$$
\begin{aligned}
& \dot{V}_{2}(t) \leqslant 2 \sum_{\iota=1}^{\kappa} e_{\iota}^{T}(t) P\left(-A e_{\iota}(t)+D g\left(Y_{\iota}(t)\right)+D g\left(Y_{0}(t)\right)+\sum_{s=1}^{m} \sum_{j=1}^{\kappa} c_{s} G_{\iota j}^{s} \Gamma_{s} e_{j}\left(t-\tau_{s}(t)\right)\right. \\
& \left.-W_{\iota} e_{\iota}(t)-\eta P^{\frac{a-1}{2}} \operatorname{sign}\left(e_{\iota}(t)\right)\left|e_{\iota}(t)\right|^{a}\right)-\sum_{s=1}^{m} c_{s} e^{T}\left(t-\tau_{s}(t)\right) Q_{s} e\left(t-\tau_{s}(t)\right) \\
& -2 \eta \sum_{\iota=1}^{\kappa} \sum_{s=1}^{m}\left(\frac{c_{s}}{1-\gamma_{s}} \int_{t-\tau_{s}(t)}^{t} e_{\iota}^{T}(h) Q_{\iota}^{s} e_{\iota}(h) d h\right)^{\frac{a+1}{2}}+\sum_{s=1}^{m} \frac{c_{s}}{1-\gamma_{s}} e^{T}(t) Q_{s} e(t) \\
& \leqslant e^{T}(t)\left[I_{\kappa} \otimes\left(-P A^{-}-A^{-} P+\varrho_{D} P^{2}+\Theta\right)\right] e(t)+\sum_{s=1}^{m} \frac{c_{s}}{1-\gamma_{s}} e^{T}(t) Q_{s} e(t) \\
& +\sum_{s=1}^{m} c_{s} e^{T}(t)\left[G^{s} \otimes\left(P \Gamma_{s}\right)\right] Q_{s}^{-1}\left[G^{s} \otimes\left(\Gamma_{s}^{T} P\right)\right] e(t)-2 \sum_{\iota=1}^{\kappa} e_{\iota}^{T}(t) P W_{\iota} e_{\iota}(t) \\
& -2 \eta \sum_{\iota=1}^{\kappa}\left(e_{\iota}^{T}(t) P e_{\iota}(t)\right)^{\frac{a+1}{2}}-2 \eta \sum_{s=1}^{m}\left(\frac{c_{s}}{1-\gamma_{s}} \int_{t-\tau_{s}(t)}^{t} e^{T}(h) Q_{s} e(h) d h\right)^{\frac{a+1}{2}} \\
& =e^{T}(t)\left\{I_{\kappa} \otimes\left(-P A^{-}-A^{-} P+\varrho_{D} P^{2}+\Theta\right)+\sum_{s=1}^{m} \frac{c_{s}}{1-\gamma_{s}} Q_{s}-\left(I_{\kappa} \otimes P\right) W\right. \\
& \left.-W^{T}\left(I_{\kappa} \otimes P\right)+\sum_{s=1}^{m} c_{s}\left[G^{s} \otimes\left(P \Gamma_{s}\right)\right] Q_{s}^{-1}\left[G^{s} \otimes\left(\Gamma_{s}^{T} P\right)\right]\right\} e(t) \\
& -2 \eta \sum_{s=1}^{m}\left(\frac{c_{s}}{1-\gamma_{s}} \int_{t-\tau_{s}(t)}^{t} e^{T}(h) Q_{s} e(h) d h\right)^{\frac{a+1}{2}} \\
& -2 \eta \sum_{\iota=1}^{\kappa}\left(e_{\iota}^{T}(t) P e_{\iota}(t)\right)^{\frac{a+1}{2}} \\
& \leqslant-2 \eta \sum_{\iota=1}^{\kappa}\left(e_{\iota}^{T}(t) P e_{\iota}(t)\right)^{\frac{a+1}{2}}-2 \eta \sum_{s=1}^{m}\left(\frac{c_{s}}{1-\gamma_{s}} \int_{t-\tau_{s}(t)}^{t} e^{T}(h) Q_{s} e(h) d h\right)^{\frac{a+1}{2}} \\
& \leqslant-2 \eta\left(\sum_{\iota=1}^{\kappa} e_{\iota}^{T}(t) P e_{\iota}(t)+\sum_{s=1}^{m} \frac{c_{s}}{1-\gamma_{s}} \int_{t-\tau_{s}(t)}^{t} e^{T}(h) Q_{s} e(h) d h\right)^{\frac{a+1}{2}} \\
& =-2 \eta\left(V_{2}(t)\right)^{\frac{a+1}{2}} .
\end{aligned}
$$

Similar to the proof of Theorem 1 , it is easy to obtain $\left\|e_{\iota}(t)\right\|_{2}=0, \iota=$ $1,2, \cdots, \kappa, t>T_{2}$. Obviously, the network (32) with the parameter ranges 
defined by (15) achieves robust finite-time anti-synchronization under the controller (24). The proof is completed.

4.2.2 Robust anti-synchronization in finite time for delayed MWCNNs with switching topology

In this subsection, the network model of delayed MWCNNs with the parameter uncertainties and switching topology is described by:

$$
\dot{Y}_{\iota}(t)=-A Y_{\iota}(t)+D g\left(Y_{\iota}(t)\right)+\sum_{s=1}^{m} \sum_{j=1}^{\kappa} c_{s} G_{\iota j}^{s, \varsigma} \Gamma_{s} Y_{j}\left(t-\tau_{s}(t)\right)+u_{\iota}(t)
$$

where $\iota=1,2, \cdots, \kappa, Y_{\iota}(t), g(\cdot), c_{s}, u_{\iota}(t), \Gamma_{s}$ represent the same definitions as those in subsection 3.1.1, $A, D$ change in some given precision described by $(15), \tau_{s}(t)$ is the time-varying delay as that in subsection 4.1.1, and $G_{\iota j}^{s, \varsigma}$ has the same meaning as that in subsection 3.1.2.

Construct the same controller as (24) for the network (35). Then,

$$
\begin{aligned}
\dot{Y}_{\iota}(t)= & -A Y_{\iota}(t)+D g\left(Y_{\iota}(t)\right)+\sum_{s=1}^{m} \sum_{j=1}^{\kappa} c_{s} G_{\iota j}^{s, \varsigma} \Gamma_{s} Y_{j}\left(t-\tau_{s}(t)\right)-W_{\iota}\left(Y_{\iota}(t)+Y_{0}(t)\right) \\
& -\eta P^{-1} \sum_{s=1}^{m}\left(\frac{c_{s}}{1-\gamma_{s}} \int_{t-\tau_{s}(t)}^{t}\left(Y_{\iota}(h)+Y_{0}(h)\right)^{T} Q_{\iota}^{s}\left(Y_{\iota}(h)+Y_{0}(h)\right) d h\right)^{\frac{a+1}{2}} \\
& \times \frac{Y_{\iota}(t)+Y_{0}(t)}{\left\|Y_{\iota}(t)+Y_{0}(t)\right\|_{2}^{2}}-\eta P^{\frac{a-1}{2}} \operatorname{sign}\left(Y_{\iota}(t)+Y_{0}(t)\right)\left|Y_{\iota}(t)+Y_{0}(t)\right|^{a} .
\end{aligned}
$$

Let $e_{\iota}(t)=Y_{\iota}(t)+Y_{0}(t)$. Then, we have

$$
\begin{aligned}
\dot{e}_{\iota}(t)= & -A e_{\iota}(t)+D g\left(Y_{\iota}(t)\right)+D g\left(Y_{0}(t)\right)+\sum_{s=1}^{m} \sum_{j=1}^{\kappa} c_{s} G_{\iota j}^{s, \varsigma} \Gamma_{s} e_{j}\left(t-\tau_{s}(t)\right)-W_{\iota} e_{\iota}(t) \\
& -\eta P^{-1} \sum_{s=1}^{m}\left(\frac{c_{s}}{1-\gamma_{s}} \int_{t-\tau_{s}(t)}^{t} e_{\iota}^{T}(h) Q_{\iota}^{s} e_{\iota}(h) d h\right)^{\frac{a+1}{2}} \frac{e_{\iota}(t)}{\left\|e_{\iota}(t)\right\|_{2}^{2}} \\
& -\eta P^{\frac{a-1}{2}} \operatorname{sign}\left(e_{\iota}(t)\right)\left|e_{\iota}(t)\right|^{a}
\end{aligned}
$$

where $\iota=1,2, \cdots, \kappa, A$ and $D$ are uncertain parameters which belong to the ranges given in (15).

Theorem 8 Suppose $\dot{\tau}_{s}(t) \leqslant \gamma_{s}<1$. The network (35) with the parameter ranges defined by (15) achieves robust finite-time anti-synchronization under the controller (24) if there exist matrices $0<P=\operatorname{diag}\left(p_{1}, p_{2}, \cdots, p_{n}\right) \in \mathbb{R}^{n \times n}$, $W=\operatorname{diag}\left(W_{1}, W_{2}, \cdots, W_{\kappa}\right) \in \mathbb{R}^{n \kappa \times n \kappa}$ and $0<Q_{s}=\operatorname{diag}\left(Q_{1}^{s}, Q_{2}^{s}, \cdots, Q_{\kappa}^{s}\right) \in$ $\mathbb{R}^{n \kappa \times n \kappa}$, such that

$$
K_{3}+K_{2}+\sum_{s=1}^{m} c_{s}\left(F_{s, \varsigma}^{T} Q_{s}^{-1} F_{s, \varsigma}+\frac{1}{1-\gamma_{s}} Q_{s}\right) \leqslant 0,
$$


where $\varsigma=1,2, \cdots, i, K_{3}=I_{\kappa} \otimes\left(-P A^{-}-A^{-} P+\varrho_{D} P^{2}+\Theta\right), K_{2}=-\left(I_{\kappa} \otimes\right.$ $P) W-W^{T}\left(I_{\kappa} \otimes P\right), \varrho_{D}=\sum_{r=1}^{n} \sum_{j=1}^{n} \tilde{d}_{r j}^{2}, F_{s, \varsigma}=G^{s, \varsigma} \otimes\left(\Gamma_{s}^{T} P\right)$. What's more, the settling time of anti-synchronization $T$ satisfies $T \leqslant T_{2}=\frac{\left(V_{2}(0)\right)^{\frac{1-a}{2}}}{\eta(1-a)}$.

Proof. Construct the same Lyapunov functional as in Theorem 5 for network (36). Then,

$$
\begin{aligned}
& D^{+} V_{2}(t) \leqslant 2 \sum_{\iota=1}^{\kappa} e_{\iota}^{T}(t) P\left(-A e_{\iota}(t)+D g\left(Y_{\iota}(t)\right)+D g\left(Y_{0}(t)\right)+\sum_{s=1}^{m} \sum_{j=1}^{\kappa} c_{s} G_{\iota j}^{s, \varsigma} \Gamma_{s} e_{j}(t\right. \\
& \left.\left.-\tau_{s}(t)\right)-W_{\iota} e_{\iota}(t)-\eta P^{\frac{a-1}{2}} \operatorname{sign}\left(e_{\iota}(t)\right)\left|e_{\iota}(t)\right|^{a}\right)+\sum_{s=1}^{m} \frac{c_{s}}{1-\gamma_{s}} e^{T}(t) Q_{s} e(t) \\
& -2 \eta \sum_{\iota=1}^{\kappa} \sum_{s=1}^{m}\left(\frac{c_{s}}{1-\gamma_{s}} \int_{t-\tau_{s}(t)}^{t} e_{\iota}^{T}(h) Q_{\iota}^{s} e_{\iota}(h) d h\right)^{\frac{a+1}{2}}-\sum_{s=1}^{m} c_{s} e^{T}(t \\
& \left.-\tau_{s}(t)\right) Q_{s} e\left(t-\tau_{s}(t)\right) \\
& \leqslant e^{T}(t)\left[I_{\kappa} \otimes\left(-P A^{-}-A^{-} P+\varrho_{D} P^{2}+\Theta\right)\right] e(t)+\sum_{s=1}^{m} \frac{c_{s}}{1-\gamma_{s}} e^{T}(t) Q_{s} e(t) \\
& +\sum_{s=1}^{m} c_{s} e^{T}(t)\left[G^{s, \varsigma} \otimes\left(P \Gamma_{s}\right)\right] Q_{s}^{-1}\left[G^{s, \varsigma} \otimes\left(\Gamma_{s}^{T} P\right)\right] e(t)-2 \sum_{\iota=1}^{\kappa} e_{\iota}^{T}(t) P W_{\iota} e_{\iota}(t) \\
& -2 \eta \sum_{\iota=1}^{\kappa}\left(e_{\iota}^{T}(t) P e_{\iota}(t)\right)^{\frac{a+1}{2}}-2 \eta \sum_{s=1}^{m}\left(\frac{c_{s}}{1-\gamma_{s}} \int_{t-\tau_{s}(t)}^{t} e^{T}(h) Q_{s} e(h) d h\right)^{\frac{a+1}{2}} \\
& =e^{T}(t)\left\{I_{\kappa} \otimes\left(-P A^{-}-A^{-} P+\varrho_{D} P^{2}+\Theta\right)+\sum_{s=1}^{m} \frac{c_{s}}{1-\gamma_{s}} Q_{s}-\left(I_{\kappa} \otimes P\right) W\right. \\
& \left.-W^{T}\left(I_{\kappa} \otimes P\right)+\sum_{s=1}^{m} c_{s}\left[G^{s, \varsigma} \otimes\left(P \Gamma_{s}\right)\right] Q_{s}^{-1}\left[G^{s, \varsigma} \otimes\left(\Gamma_{s}^{T} P\right)\right]\right\} e(t) \\
& -2 \eta \sum_{s=1}^{m}\left(\frac{c_{s}}{1-\gamma_{s}} \int_{t-\tau_{s}(t)}^{t} e^{T}(h) Q_{s} e(h) d h\right)^{\frac{a+1}{2}} \\
& -2 \eta \sum_{\iota=1}^{\kappa}\left(e_{\iota}^{T}(t) P e_{\iota}(t)\right)^{\frac{a+1}{2}} \\
& \leqslant-2 \eta \sum_{\iota=1}^{\kappa}\left(e_{\iota}^{T}(t) P e_{\iota}(t)\right)^{\frac{a+1}{2}}-2 \eta \sum_{s=1}^{m}\left(\frac{c_{s}}{1-\gamma_{s}} \int_{t-\tau_{s}(t)}^{t} e^{T}(h) Q_{s} e(h) d h\right)^{\frac{a+1}{2}} \\
& \leqslant-2 \eta\left(\sum_{\iota=1}^{\kappa} e_{\iota}^{T}(t) P e_{\iota}(t)+\sum_{s=1}^{m} \frac{c_{s}}{1-\gamma_{s}} \int_{t-\tau_{s}(t)}^{t} e^{T}(h) Q_{s} e(h) d h\right)^{\frac{a+1}{2}} \\
& =-2 \eta\left(V_{2}(t)\right)^{\frac{a+1}{2}} \text {. }
\end{aligned}
$$


Similar to the proof of Theorem 1 , it is easy to obtain $\left\|e_{\iota}(t)\right\|_{2}=0, \iota=$ $1,2, \cdots, \kappa, t>T_{2}$. Obviously, the network (35) with the parameter ranges defined by (15) achieves robust finite-time anti-synchronization under the controller (24). The proof is completed.

Remark 2 In this paper, by designing suitable state feedback controllers, several sufficient conditions which guarantee the finite-time anti-synchronization of MWCNNs with and without coupling delays are obtained. To the best of knowledge, this is the first paper toward to studying anti-synchronization for MWCNNs in finite time. Recently, a few scholars established some novel finitetime synchronization criteria for complex networks via intermittent feedback control scheme $[35,36]$. Incorporating this new control approach and techniques into the research on finite-time anti-synchronization of MWCNNs is a very interesting and challenging problem, which would become one of research topics of our future work.

\section{Numerical Examples}

Example 4.1. Consider the switched MWCNNs with parameter uncertainties as follows:

$$
\begin{aligned}
\dot{Y}_{\iota}(t)= & -A Y_{\iota}(t)+D g\left(Y_{\iota}(t)\right)+0.5 \sum_{j=1}^{6} G_{\iota j}^{1, \varsigma} \Gamma_{1} Y_{j}(t)+0.8 \sum_{j=1}^{6} G_{\iota j}^{2, \varsigma} \Gamma_{2} Y_{j}(t) \\
& +1.1 \sum_{j=1}^{6} G_{\iota j}^{3, \varsigma} \Gamma_{3} Y_{j}(t)+u_{\iota}(t),
\end{aligned}
$$

where $\iota=1,2, \cdots, 6, \varsigma=1,2, g_{k}(\sigma)=\frac{|\sigma+1|-|\sigma-1|}{4}(k=1,2,3), G^{1,1}$ and $G^{1,2}$ are two possible topologies which are switched as $G^{1,1} \rightarrow G^{1,2} \rightarrow G^{1,1} \rightarrow$ $G^{1,2} \rightarrow \cdots$, and each graph is active for $1 \mathrm{~s}, G^{2,1}, G^{2,2}, G^{3,1}, G^{3,2}$ are switched in the same way. The inner coupling matrices $\Gamma_{1}, \Gamma_{2}, \Gamma_{3}$ and the coupling matrices $G^{1,1}=\left(G_{\iota j}^{1,1}\right)_{6 \times 6}, G^{1,2}=\left(G_{\iota j}^{1,2}\right)_{6 \times 6}, G^{2,1}=\left(G_{\iota j}^{2,1}\right)_{6 \times 6}, G^{2,2}=$ 
$\left(G_{\iota j}^{2,2}\right)_{6 \times 6}, G^{3,1}=\left(G_{\iota j}^{3,1}\right)_{6 \times 6}, G^{3,2}=\left(G_{\iota j}^{3,2}\right)_{6 \times 6}$ are chosen as, respectively

$$
\begin{aligned}
& \Gamma_{1}=\left(\begin{array}{ccc}
0.6 & 0.1 & 0 \\
0 & 0.4 & 0 \\
0 & 0.2 & 0.5
\end{array}\right), \Gamma_{2}=\left(\begin{array}{ccc}
0.7 & 0 & 0.2 \\
0 & 0.4 & 0 \\
0.3 & 0 & 0.7
\end{array}\right), \Gamma_{3}=\left(\begin{array}{ccc}
0.2 & 0 & 0 \\
0 & 0.7 & 0.2 \\
0.1 & 0 & 0.6
\end{array}\right), \\
& G^{1,1}=\left(\begin{array}{cccccc}
-0.7 & 0.1 & 0.1 & 0.3 & 0.2 & 0 \\
0.1 & -0.8 & 0 & 0.2 & 0.2 & 0.3 \\
0.1 & 0 & -0.4 & 0.2 & 0 & 0.1 \\
0.3 & 0.2 & 0.2 & -1.2 & 0.3 & 0.2 \\
0.2 & 0.2 & 0 & 0.3 & -0.9 & 0.2 \\
0 & 0.3 & 0.1 & 0.2 & 0.2 & -0.8
\end{array}\right) \text {, } \\
& G^{1,2}=\left(\begin{array}{cccccc}
-0.6 & 0.2 & 0 & 0.2 & 0.1 & 0.1 \\
0.2 & -0.5 & 0.1 & 0.1 & 0.1 & 0 \\
0 & 0.1 & -0.7 & 0 & 0.4 & 0.2 \\
0.2 & 0.1 & 0 & -0.5 & 0.2 & 0 \\
0.1 & 0.1 & 0.4 & 0.2 & -0.9 & 0.1 \\
0.1 & 0 & 0.2 & 0 & 0.1 & -0.4
\end{array}\right) \text {, } \\
& G^{2,1}=\left(\begin{array}{cccccc}
-0.8 & 0.2 & 0.1 & 0.2 & 0.3 & 0 \\
0.2 & -0.6 & 0 & 0.2 & 0 & 0.2 \\
0.1 & 0 & -1.0 & 0.3 & 0.4 & 0.2 \\
0.2 & 0.2 & 0.3 & -1.2 & 0.1 & 0.4 \\
0.3 & 0 & 0.4 & 0.1 & -1.0 & 0.2 \\
0 & 0.2 & 0.2 & 0.4 & 0.2 & -1.0
\end{array}\right) \\
& G^{2,2}=\left(\begin{array}{cccccc}
-0.5 & 0.1 & 0 & 0.1 & 0.1 & 0.2 \\
0.1 & -0.7 & 0.4 & 0.1 & 0.1 & 0 \\
0 & 0.4 & -1.2 & 0 & 0.5 & 0.3 \\
0.1 & 0.1 & 0 & -0.5 & 0.3 & 0 \\
0.1 & 0.1 & 0.5 & 0.3 & -1.1 & 0.1 \\
0.2 & 0 & 0.3 & 0 & 0.1 & -0.6
\end{array}\right) \text {, } \\
& G^{3,1}=\left(\begin{array}{cccccc}
-0.7 & 0.3 & 0.1 & 0 & 0.1 & 0.2 \\
0.3 & -0.9 & 0.3 & 0.1 & 0 & 0.2 \\
0.1 & 0.3 & -0.9 & 0.2 & 0.3 & 0 \\
0 & 0.1 & 0.2 & -0.8 & 0.4 & 0.1 \\
0.1 & 0 & 0.3 & 0.4 & -0.9 & 0.1 \\
0.2 & 0.2 & 0 & 0.1 & 0.1 & -0.6
\end{array}\right) \text {, } \\
& G^{3,2}=\left(\begin{array}{cccccc}
-0.6 & 0.2 & 0 & 0.1 & 0.2 & 0.1 \\
0.2 & -0.8 & 0.2 & 0.2 & 0.2 & 0 \\
0 & 0.2 & -0.7 & 0 & 0.1 & 0.4 \\
0.1 & 0.2 & 0 & -0.6 & 0.3 & 0 \\
0.2 & 0.2 & 0.1 & 0.3 & -1.0 & 0.2 \\
0.1 & 0 & 0.4 & 0 & 0.2 & -0.7
\end{array}\right) \text {. }
\end{aligned}
$$


The parameters $A=\operatorname{diag}\left(a_{1}, a_{2}, \cdots, a_{n}\right), D=\left(d_{r j}\right)_{n \times n}$ in the network (38) can be changed in the following given precisions:

$$
\left\{\begin{aligned}
A_{I}:= & \left\{A=\operatorname{diag}\left(a_{r}\right): A^{-} \leqslant A \leqslant A^{+}, \text {i.e., } 0<0.5 r \leqslant a_{r} \leqslant 0.6 r\right. \\
& \left.r=1,2, \cdots, n, \forall A \in A_{I}\right\}, \\
D_{I}:= & \left\{D=\left(d_{r j}\right)_{n \times n}: D^{-} \leqslant D \leqslant D^{+}, i . e ., \frac{0.2 j}{r+j} \leqslant d_{r j} \leqslant \frac{0.3 j}{r+j},\right. \\
& \left.r, j=1,2, \cdots, n, \forall D \in D_{I}\right\} .
\end{aligned}\right.
$$

Obviously, $g_{k}(\cdot)(k=1,2,3)$ satisfies Assumption 1 with $\theta_{k}=0.5$. Take $W=\operatorname{diag}\left(0.2 I_{3}, 0.4 I_{3}, 0.3 I_{3}, 0.6 I_{3}, 0.7 I_{3}, 0.5 I_{3}\right)$. The following matrix $P$ satisfying (22) can be computed by utilizing MATLAB Toolbox:

$$
P=\left(\begin{array}{ccc}
0.3252 & 0 & 0 \\
0 & 0.2505 & 0 \\
0 & 0 & 0.1992
\end{array}\right)
$$

According to Theorem 4, the network (38) with the parameter ranges defined by (39) achieves robust finite-time anti-synchronization under the controller (3) and the time estimation of achieving anti-synchronization is $T_{1}=9.32$. The simulation result is displayed in Figure 1 .

Example 4.2. Consider the following switched MWCNNs with the parameter uncertainties and coupling delays:

$$
\begin{aligned}
\dot{Y}_{\iota}(t)= & -A Y_{\iota}(t)+D g\left(Y_{\iota}(t)\right)+0.2 \sum_{j=1}^{6} G_{\iota j}^{1, \varsigma} \Gamma_{1} Y_{j}\left(t-\tau_{1}(t)\right)+0.1 \sum_{j=1}^{6} G_{\iota j}^{2, \varsigma} \Gamma_{2} Y_{j}(t \\
& \left.-\tau_{2}(t)\right)+0.3 \sum_{j=1}^{6} G_{\iota j}^{3, \varsigma} \Gamma_{3} Y_{j}\left(t-\tau_{3}(t)\right)+u_{\iota}(t)
\end{aligned}
$$

where $\iota=1,2, \cdots, 6, \varsigma=1,2, g_{k}(\sigma)=\frac{|\sigma+1|-|\sigma-1|}{8}(k=1,2,3), \tau_{1}(t)=$ $\frac{1}{10}-\frac{1}{30} e^{-t}, \tau_{2}(t)=\frac{1}{10}-\frac{1}{40} e^{-t}, \tau_{3}(t)=\frac{1}{10}-\frac{1}{50} e^{-t}, \tau=\frac{1}{10}, \gamma_{1}=\frac{1}{30}, \gamma_{2}=$ $\frac{1}{40}, \gamma_{3}=\frac{1}{50}, G^{1,1}$ and $G^{1,2}$ are two possible topologies which are switched as $G^{1,1} \rightarrow G^{1,2} \rightarrow G^{1,1} \rightarrow G^{1,2} \rightarrow \cdots$, and $G^{2,1}, G^{2,2}, G^{3,1}, G^{3,2}$ are switched in the same way with activation time of $1 \mathrm{~s}$ for each graph. The inner coupling matrices $\Gamma_{1}, \Gamma_{2}, \Gamma_{3}$ and the coupling matrices $G^{1,1}=\left(G_{\iota j}^{1,1}\right)_{6 \times 6}, G^{1,2}=$ $\left(G_{\iota j}^{1,2}\right)_{6 \times 6}, G^{2,1}=\left(G_{\iota j}^{2,1}\right)_{6 \times 6}, G^{2,2}=\left(G_{\iota j}^{2,2}\right)_{6 \times 6}, G^{3,1}=\left(G_{\iota j}^{3,1}\right)_{6 \times 6}, G^{3,2}=$ $\left(G_{\iota j}^{3,2}\right)_{6 \times 6}$ are chosen as, respectively 


$$
\begin{aligned}
& \Gamma_{1}=\left(\begin{array}{ccc}
0.4 & 0.1 & 0 \\
0 & 0.5 & 0 \\
0 & 0.2 & 0.6
\end{array}\right), \Gamma_{2}=\left(\begin{array}{ccc}
0.6 & 0 & 0.3 \\
0 & 0.2 & 0 \\
0.1 & 0 & 0.4
\end{array}\right), \Gamma_{3}=\left(\begin{array}{ccc}
0.3 & 0 & 0 \\
0.1 & 0.4 & 0.2 \\
0 & 0 & 0.5
\end{array}\right) \\
& G^{1,1}=\left(\begin{array}{cccccc}
-0.5 & 0.2 & 0.1 & 0 & 0.1 & 0.1 \\
0.2 & -1.0 & 0.2 & 0.3 & 0.1 & 0.2 \\
0.1 & 0.2 & -0.6 & 0.2 & 0 & 0.1 \\
0 & 0.3 & 0.2 & -0.9 & 0.3 & 0.1 \\
0.1 & 0.1 & 0 & 0.3 & -0.5 & 0 \\
0.1 & 0.2 & 0.1 & 0.1 & 0 & -0.5
\end{array}\right) \\
& G^{1,2}=\left(\begin{array}{cccccc}
-1.0 & 0.3 & 0 & 0.2 & 0.3 & 0.2 \\
0.3 & -0.8 & 0.1 & 0.2 & 0.2 & 0 \\
0 & 0.1 & -0.8 & 0 & 0.5 & 0.2 \\
0.2 & 0.2 & 0 & -0.5 & 0.1 & 0 \\
0.3 & 0.2 & 0.5 & 0.1 & -1.4 & 0.3 \\
0.2 & 0 & 0.2 & 0 & 0.3 & -0.7
\end{array}\right) \text {, } \\
& G^{2,1}=\left(\begin{array}{cccccc}
-0.9 & 0.3 & 0.2 & 0 & 0.3 & 0.1 \\
0.3 & -1.0 & 0 & 0.3 & 0.1 & 0.3 \\
0.2 & 0 & -0.8 & 0.1 & 0.5 & 0 \\
0 & 0.3 & 0.1 & -0.7 & 0.1 & 0.2 \\
0.3 & 0.1 & 0.5 & 0.1 & -1.0 & 0 \\
0.1 & 0.3 & 0 & 0.2 & 0 & -0.6
\end{array}\right) \text {, } \\
& G^{2,2}=\left(\begin{array}{cccccc}
-0.7 & 0.2 & 0 & 0.2 & 0.1 & 0.2 \\
0.2 & -0.8 & 0.2 & 0.2 & 0.2 & 0 \\
0 & 0.2 & -0.8 & 0 & 0.4 & 0.2 \\
0.2 & 0.2 & 0 & -0.7 & 0.3 & 0 \\
0.1 & 0.2 & 0.4 & 0.3 & -1.3 & 0.3 \\
0.2 & 0 & 0.2 & 0 & 0.3 & -0.7
\end{array}\right) \text {, } \\
& G^{3,1}=\left(\begin{array}{cccccc}
-0.7 & 0.1 & 0.1 & 0.3 & 0.2 & 0 \\
0.1 & -0.8 & 0.2 & 0 & 0.3 & 0.2 \\
0.1 & 0.2 & -1.0 & 0.2 & 0.3 & 0.2 \\
0.3 & 0 & 0.2 & -0.6 & 0 & 0.1 \\
0.2 & 0.3 & 0.3 & 0 & -0.9 & 0.1 \\
0 & 0.2 & 0.2 & 0.1 & 0.1 & -0.6
\end{array}\right), \\
& G^{3,2}=\left(\begin{array}{cccccc}
-0.8 & 0.2 & 0 & 0.4 & 0.1 & 0.1 \\
0.2 & -0.8 & 0.3 & 0.2 & 0.1 & 0 \\
0 & 0.3 & -0.8 & 0 & 0.4 & 0.1 \\
0.4 & 0.2 & 0 & -0.8 & 0.2 & 0 \\
0.1 & 0.1 & 0.4 & 0.2 & -1.0 & 0.2 \\
0.1 & 0 & 0.1 & 0 & 0.2 & -0.4
\end{array}\right) \text {. }
\end{aligned}
$$

The parameters $A=\operatorname{diag}\left(a_{1}, a_{2}, \cdots, a_{n}\right), D=\left(d_{r j}\right)_{n \times n}$ in the network (40) can be changed same as those in (39). Obviously, $g_{k}(\cdot)(k=1,2,3)$ satisfies 


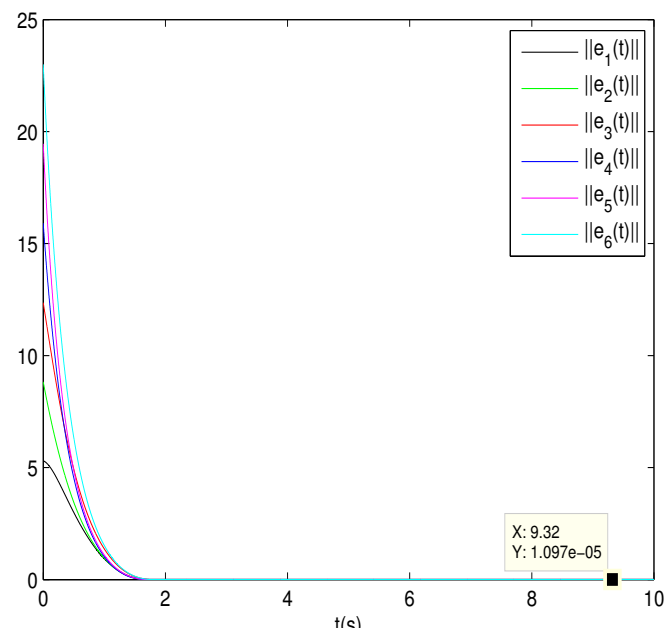

Fig. $1\left\|e_{\iota}(t)\right\|, \iota=1,2, \cdots, 6$

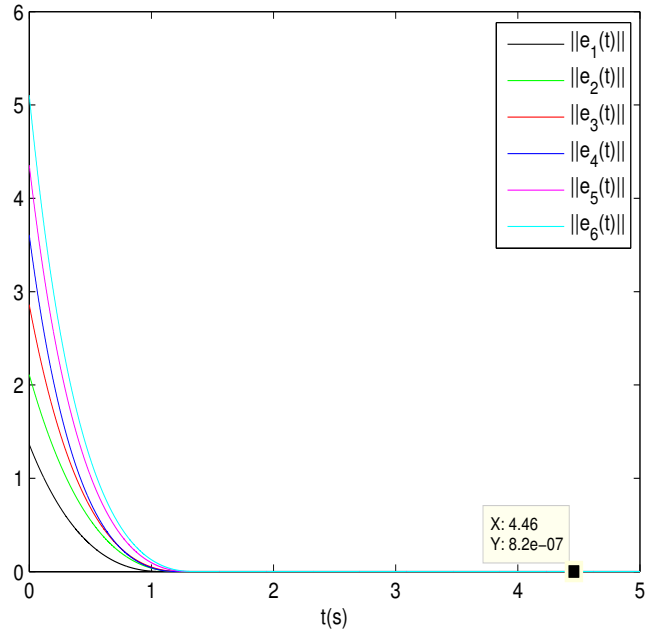

Fig. $2\left\|e_{\iota}(t)\right\|, \iota=1,2, \cdots, 6$.

Assumption 1 with $\theta_{k}=0.25$. Take $W=\operatorname{diag}\left(0.2 I_{3}, 0.1 I_{3}, 0.3 I_{3}, 0.6 I_{3}, 0.4 I_{3}\right.$, $\left.0.5 I_{3}\right)$. By employing MATLAB Toolbox, the following matrices $P, Q_{1}, Q_{2}$ 
and $Q_{3}$ satisfying (37) can be obtained:

$$
\begin{aligned}
P & =\left(\begin{array}{ccc}
0.4613 & 0 & 0 \\
0 & 0.3981 & 0 \\
0 & 0 & 0.3020
\end{array}\right), \\
Q_{1} & =I_{6} \otimes\left(\begin{array}{ccc}
0.2083 & 0 & 0 \\
0 & 0.2107 & 0 \\
0 & 0 & 0.2110
\end{array}\right), \\
Q_{2} & =I_{6} \otimes\left(\begin{array}{ccc}
0.1050 & 0 & 0 \\
0 & 0.1053 & 0 \\
0 & 0 & 0.1054
\end{array}\right), \\
Q_{3} & =I_{6} \otimes\left(\begin{array}{ccc}
0.3067 & 0 & 0 \\
0 & 0.3145 & 0 \\
0 & 0 & 0.3157
\end{array}\right) .
\end{aligned}
$$

According to Theorem 8, the network (40) with the parameter ranges defined by (39) achieves robust finite-time anti-synchronization under the controller (24) and the time estimation of achieving anti-synchronization is $T_{2}=4.46$. The simulation result is displayed in Figure 2 .

\section{Conclusion}

In this paper, the finite-time anti-synchronization and robust finite-time antisynchronization for MWCNNs with and without coupling delays have been respectively studied. By making use of Lyapunov functional method as well as inequality techniques, some finite-time anti-synchronization and robust finitetime anti-synchronization conditions have been derived for those network models. Furthermore, we have also considered the finite-time anti-synchronization and robust finite-time anti-synchronization of MWCNNs with switching topology. Finally, several simulation examples have been provided to verified the correctness of our results.

\section{Acknowledgement}

The authors would like to thank the Associate Editor and anonymous reviewers for their valuable comments and suggestions. They also wish to express their sincere appreciation to Prof. Jinliang Wang for the fruitful discussions and valuable suggestions which helped to improve this paper. This work was supported in part by the Natural Science Foundation of Tianjin under Grant 18JCQNJC74300, in part by China Scholarship Council (No.201808120044), and in part by the National Natural Science Foundation of China under Grant 61773285. Dr E. Yang is supported in part under the RSE-NNSFC Joint Project (2017-2019) (6161101383) with China University of Petroleum (East China). 


\section{References}

1. Lin SH, Kung SY, Lin LJ (1997) Face recognition/detection by probabilistic decisionbased neural network. IEEE Trans Neural Netw 8(1): 114-132

2. Asadia E, Silva MGda, Antunes CH, Diasc L, Glicksman L (2014) Multi-objective optimization for building retrofit: a model using genetic algorithm and artificial neural network and an application. Energ Buildings 81: 444-456

3. Sakthivel R, Vadivel P, Mathiyalaganc K, Arunkumar A, Sivachitra M (2015) Design of state estimator for bidirectional associative memory neural networks with leakage delays. Inform Sciences 296:263-274

4. Zheng ZW, Huang YT, Xie LH, Zhu B (2018) Adaptive trajectory tracking control of a fully actuated surface vessel with asymmetrically constrained input and output. IEEE Trans Control Syst Technol 26(5): 1851-1859

5. Zheng ZW, Sun L, Xie LH (2018) Error constrained LOS path following of a surface vessel with actuator saturation and faults. IEEE Trans Syst Man Cyber: Syst 48(10): $2168-2216$

6. Gao F, Huang T, Sun JP, Wang J, Hussain A, Yang E (2018) A new algorithm of SAR image target recognition based on improved deep convolutional neural network. Cogn Comput http://doi.rog/10.1007/s12559-018-9563-Z

7. Yue ZY, Gao F, Xiong QX, Wang J, Huang T, Yang E, Zhou HY (2019) A novel semi-supervised convolutional neural network method for synthetic aperture radar image recognition. Cogn Comput http://doi.rog/10.1007/s12559-019-09639-x

8. Zhang CK, He Y, Jiang L, Wang QG, Wu M (2017) Stability analysis of discrete-time neural networks with time-varying delay via an extended reciprocally convex matrix inequality. IEEE Trans Cybern 47(10): 3040-3049

9. Wang H, Duan S, Huang T, Wang L, Li C (2017) Exponential stability of complex-valued memristive recurrent neural networks. IEEE Trans Neural Netw Learn Syst 28(3): 766771

10. Wang JL, Wu HN, Guo L (2011) Passivity and stability analysis of reaction-diffusion neural networks with dirichlet boundary conditions. IEEE Trans Neural Netw 22(12): 2105-2116

11. Yang CB, Huang TZ (2014) Improved stability criteria for a class of neural networks with variable delays and impulsive perturbations. Appl Math Comput 243: 923-935

12. Ahn CK (2013) Passive and exponential filter design for fuzzy neural networks. Inform Sciences 238: 126-137

13. Wang LM, Zeng ZG, Ge MF, Hu JH (2018) Global stabilization analysis of inertial memristive recurrent neural networks with discrete and distributed delays. Neural Netw 105: 65-74

14. Prakash M, Balasubramaniam P, Lakshmanan S (2016) Synchronization of Markovian jumping inertial neural networks and its applications in image encryption. Neural Netw 83: 86-93

15. Xie QX, Chen GR, Bollt EM (2002) Hybrid chaos synchronization and its application in information processing. Math Comput Model 35: 145-163

16. Zhang J, Gao YB (2017) Synchronization of coupled neural networks with time-varying delay. Neurocomputing 219: 154-162

17. Zhang H, Sheng Y, Zeng ZG (2018) Synchronization of coupled reaction-diffusion neural networks with directed topology via an adaptive approach. IEEE Trans Neural Netw Learn Syst 29(5): 1550-1561

18. Wang JY, Zhang HG, Wang ZS, Shan QH (2016) Local synchronization criteria of markovian nonlinearly coupled neural networks with uncertain and partially unknown transition rates. IEEE Trans Syst Man Cybern 47(8): 1953-1964

19. Bao HB, Park JH, Cao JD (2016) Exponential synchronization of coupled stochastic memristor-based neural networks with time-varying probabilistic delay coupling and impulsive delay. IEEE Trans Neural Netw Learn Syst 27(1): 190-201

20. Chen HB, Shi P, Lim CC (2017) Exponential synchronization for markovian stochastic coupled neural networks of neutral-type via adaptive feedback control. IEEE Trans Neural Netw Learn Syst 28(7): 1618-1632 
21. Huang YL, Xu BB, Ren SY (2018) Analysis and pinning control for passivity of coupled reaction-diffusion neural networks with nonlinear coupling. Neurocomputing 272(10): 334-342

22. Huang YL, Wang SX, Ren SY (2019) Pinning exponential synchronization and passivity of coupled delayed reaction-diffusion neural networks with and without parametric uncertainties. Int J Control 92(5): 1167-1182

23. Altafini C (2013) Consensus problems on networks with antagonistic interactions. IEEE Trans Automa Control 58(4): 935-946

24. Kim CM, Rim S, Kye WH, Ryu JW, Park YJ (2003) Anti-synchronization of chaotic oscillators. Phys Lett A 320(1): 39-46

25. Wang WP, Li LX, Peng HP, Wang WN, Kurths J, Xiao JH, Yang YX (2016) Antisynchronization of coupled memristive neutral-type neural networks with mixed timevarying delays via randomly occurring control. Nonlinear Dyn 83(4): 2143-2155

26. Liu D, Zhu S, Sun K (2018) Global anti-synchronization of complexvalued memristive neural networks with time delays. IEEE Trans Cybern https://doi.org/10.1109/TCYB.2018.2812708

27. Zhao HY, Zhang Q (2011) Global impulsive exponential anti-synchronization of delayed chaotic neural networks. Neurocomputing 74: 563-567

28. Sakthivel R, Anbuvithya R, Mathiyalagan K, Ma YK, Prakash P (2016) Reliable antisynchronization conditions for BAM memristive neural networks with different memductance functions. Appl Math Comput 275: 213-228

29. Wang JY, Zhang HG, Wang ZS, Gao WZ (2017) Finite-time synchronization of coupled hierarchical hybrid neural networks with time-varying delays. IEEE Trans Cybern 47(10): 2995-3004

30. Liu XY, Cao JD, Yu WW, Song Q (2016) Nonsmooth finite-time synchronization of switched coupled neural networks. IEEE Trans Cybern 46(10): 2360-2371

31. Liu XW, Chen TP (2018) Finite-time and fixed-time cluster synchronization with or without pinning control. IEEE Trans Cybern 48(1): 240-252

32. Sun JW, Wang Y, Wang YF, Shen Y (2016) Finite-time synchronization between two complex-variable chaotic systems with unknown parameters via nonsingular terminal sliding mode control. Nonlinear Dyn 85(2): 1105-1117

33. Sun JW, Wu YY, Cui GZ, Wang YF (2017) Finite-time real combination synchronization of three complex-variable chaotic systems with unknown parameters via sliding mode control. Nonlinear Dyn 88(3): 1677-1690

34. Sun JW, Zhao XT, Fang J, Wang YF (2018) Autonomous memristor chaotic systems of infinite chaotic attractors and circuitry realization. Nonlinear Dyn 94(4): 2879-2887

35. Zhang DY, Shen YJ, Mei J (2017) Finite-time synchronization of multi-layer nonlinear coupled complex networks via intermittent feedback control. Neurocomputing 225: 129138

36. Mei J, Jiang MH, Xu WM, Wang B (2013) Finite-time synchronization control of complex dynamical networks with time delay. Commun Nonlinear Sci Numer Simulat 18(9): $2462-2478$

37. Wang LM, Shen Y, Zhang GD (2017) Finite-time stabilization and adaptive control of memristor-based delayed neural networks. IEEE Trans Neural Netw Learn Syst 28(11): 2648-2659

38. Wang LM, Zeng ZG, Hu JH, Wang XP (2017) Controller design for global fixed-time synchronization of delayed neural networks with discontinuous activations. Neural Netw 87: $122-131$

39. Huang YL, Qiu SH, Ren SY (2019) Finite-time synchronization and passivity of coupled memristive neural networks. Int $\mathrm{J}$ Control http://doi.rog/10.1080/00207179.2019.1566640

40. Zheng ZW, Xie LH (2017) Finite-time path following control for a stratospheric airship with input saturation and error constraint. Int $\mathrm{J}$ Control http://doi.rog/10.1080/00207179.2017.1357839

41. Sui X, Yang YQ, Wang F, Zhang LZ (2017) Finite-time anti-synchronization of timevarying delayed neural networks via feedback control with intermittent adjustment. Adv Differ Equ-NY https://doi.org/10.1186/s13662-017-1264-5 
42. Wang WP, Li LX, Peng HP, Kurths J, Xiao JH, Yang YX (2016) Finite-time antisynchronization control of memristive neural networks with stochastic perturbations. Neural Process Lett 43(1): 49-63

43. Qiu SH, Huang YL, Ren SY (2018) Finite-time synchronization of multi-weighted complex dynamical networks with and without coupling delay. Neurocomputing 275:12501260

44. Wang JL, Xu M, Wu HN, Huang TW (2018) Finite-time passivity of coupled neural networks with multiple weights. IEEE Trans Network Sci Eng 5(3): 184-197

45. Huang YL, Ren SY (2018) Passivity and passivity-based synchronization of switched coupled reaction-diffusion neural networks with state and spatial diffusion couplings. Neural Process Lett 47(2): 347-363

46. Wu YY, Cao JD, Li QB, Alsaedi A, Alsaadi FE (2017) Finite-time synchronization of uncertain coupled switched neural networks under asynchronous switching. Neural Netw 85: $128-139$

47. Wen GH, Yu WW, Hu GQ, Cao JD, Yu XH, Pinning synchronization of directed networks with switching topologies: a multiple lyapunov functions approach. IEEE Trans Neural Netw Learn Syst 26(12): 3239-3250

48. Zhen Q, Wang JL, Huang YL, Ren SY (2017) Synchronization and $\mathcal{H}_{\infty}$ synchronization of multi-weighted complex delayed dynamical networks with fixed and switching topologies. J Franklin Inst 354: 7119-7138

49. Wang SX, Huang YL, Ren SY (2017) Synchronization and robust synchronization for fractional-order coupled neural networks. IEEE Access 5: 12439-12448

50. Zheng C, Cao JD (2014) Robust synchronization of coupled neural networks with mixed delays and uncertain parameters by intermittent pinning control. Neurocomputing 141:153-159

51. Qin Z, Wang JL, Huang YL, Ren SY (2018) Analysis and adaptive control for robust synchronization and $\mathcal{H}_{\infty}$ synchronization of complex dynamical networks with multiple time-delays. Neurocomputing 289: 241-251

52. Liang JL, Wang ZD, Liu YR, Liu XH (2008) Robust Synchronization of an array of coupled stochastic discrete-time delayed neural networks. IEEE Trans Neural Netw 19(11): 1910-1921

53. Tang Y (1998) Terminal sliding mode control for rigid robots. Automatica 34(1d): 51-56

54. Huang XQ, Lin W, Yang B (2005) Global finite-time stabilization of a class of uncertain nonlinear systems. Automatica 41: 881-888

55. Shen H, Huo SC, Cao JD, Huang TW (2019) Generalized state estimation for Markovian coupled networks under round-robin protocol and redundant channels. IEEE Trans Cybern 49(4): 1292-1301

56. Shen H, Li F, Yan HC, Karimi HR, Lam HK (2019) Finite-time event-triggered $\mathcal{H}_{\infty}$ control for T-S fuzzy Markov jump systems. IEEE Trans Fuzzy Syst 26(5): 3122-3135

57. Shen H, Li F, Xu SY, Sreeram V (2018) Slow state variables feedback stabilization for semi-Markov jump systems with singular perturbations. IEEE Trans Automa Control 63(8): 2709-2714

58. Shen H, Wang T, Cao JD, Lu GP, Song YD, Huang TW (2018) Nonfragile dissipative synchronization for Markovian Memristive neural networks: a gain-scheduled control scheme. IEEE Trans Neural Netw Learn Syst http://doi.rog/10.1109/TNNLS.2018.2874035

59. Shen H, Men YZ, Wu ZG, Cao JD, Lu GP (2019) Network-based quantized control for fuzzy singularly perturbed semi-Markov jump systems and its application. IEEE Trans Circuits Syst I Regul Pap 66(3): 1130-1140

60. Shen H, Li F, Wu ZG, Park JH, Sreeram V (2018) Fuzzy-model-based non-fragile control for nonlinear singularly perturbed systems with semi-Markov jump parameters. IEEE Trans Fuzzy Syst. 26(6): 3428-3439

61. Shen H, Men YZ, Wu ZG, Park JH (2018) Nonfragile $\mathcal{H}_{\infty}$ control for fuzzy Markovian jump systems under fast sampling singular perturbation. IEEE Trans Syst Man Cyber Syst 48(12): 2058-2069 